\title{
Toward Understanding of the Role of Reversibility of Phenotypic Switching in the Evolution of Resistance to Therapy.
}

\author{
D. Horvath ${ }^{1}$ and B. Brutovsky ${ }^{2}$ \\ ${ }^{1}$ Center of Interdisciplinary Biosciences, Technology and Innovation Park, P. J. Šafárik \\ University, Jesenná 5, 04154 Košice, Slovak Republic \\ ${ }^{2}$ Department of Biophysics, Faculty of Science, Jesenná 5, P. J. Šafárik University, 04154 \\ Košice, Slovak Republic
}

\begin{abstract}
Reversibility of state transitions is intensively studied topic in many scientific disciplines over many years. In cell biology, it plays an important role in epigenetic variation of phenotypes, known as phenotypic plasticity. More interestingly, the cell state reversibility is probably crucial in the adaptation of population phenotypic heterogeneity to environmental fluctuations by evolving bet-hedging strategy, which might confer to cancer cells resistance to therapy. In this article, we propose a formalization of the evolution of highly reversible states in the environments of periodic variability. Two interrelated models of heterogeneous cell populations are proposed and their behavior is studied. The first model captures selection dynamics of the cell clones for the respective levels of phenotypic reversibility. The second model focuses on the interplay between reversibility and drug resistance in the particular case of cancer. Overall, our results show that the threshold dependencies are emergent features of the investigated model with eventual therapeutic relevance. Presented examples demonstrate importance of taking into account cell to cell heterogeneity within a system of clones with different reversibility quantified by appropriately chosen genetic and epigenetic entropy measures.
\end{abstract}

\section{Introduction}

Human diseases are typically caused by invading pathogenic microorganisms, such as viruses, bacteria, fungi, prions, etc. Despite immunity system is able to cope with the majority of pathogens, those escaping from innate immunity surveillance must be treated by therapy. The main obstacle to efficient therapy is the variability of the microorganisms within the population, such as strain or clone, conferring them resistance to therapy. Population variability flows from the evolutionary essence of population dynamics of microorganisms which equips evolving populations of pathogens with powerful adaptive capability. For example, evolutionary dynamics of carcinogenesis [1, 2, 3] is considered the main reason why targeted therapy does not work [4].

As it is known for a long time, intratumor heterogeneity is not bound exclusively to the differences in DNA sequences of the respective cells (genetic heterogeneity), but to the epigenetic differences as well [5]. It is broadly accepted that the epigenetic mechanisms for gene transcription regulation are usually reversible [6]. It has been found that the role of epigenetic mechanisms, such as DNA methylation, histone modifications, chromatin remodeling, and small RNA molecules, in cancer initiation and progression is causative [7, 8]. In particular, variability in phenotypic characteristics of isogenic cells, known as phenotypic plasticity, is assumed to be an important cause of the therapeutic resilience of advanced cancers [9].

Recognizing tumor dynamics as evolutionary process, one can exploit known universal features of evolution to influence the evolution of the population in desirable direction in mathematically more purposeful way. While the role of genetic intratumor heterogeneity in tumor evolution was accepted long time ago, the role of epigenetic hetero- 
geneity is much less obvious. To implement evolutionary principles into the therapy design, the interplay between genetic diversity and epigenetic plasticity [10] should be carefully studied at the model level within an evolutionary-based integrative conceptual framework. Recently, the concept of Waddington's epigenetic landscape was applied to formalize the relation between the cancer cell genome and epigenetic mechanisms [11] in mathematically instructive way. Therein, each point in the fitness landscape (i. e. genome) provides epigenetic landscape of unique topology. Due to their mathematical complexity, the epigenetic landscapes contain many areas (attractors) around the stable cell-states corresponding to the respective stable phenotypes of a cell. Straightforwardly, the phenotypic switching corresponds to the transition between two such attractors.

Below we use the above conceptualization [11] as an instructive backbone for our considerations. As different cell states in the epigenetic landscape differ in their fitness-related properties, the cell states composition (or non-genetic heterogeneity) becomes, from the viewpoint of the clone, evolutionary important at the clone's respective timescale. It was observed that in the case of variable selective pressure, population of organisms evolve mechanisms to tune the phenotypic variability to the variability of the acting selective pressure [12]. In bacteria, the well known riskdiversification strategy evolved in the populations when facing uncertain future and/or environment $[13,14,15]$ is the bet-hedging strategy [16, 17, 10]. Based on formal similarity of evolving cancer cells population with bacteria, viruses or yeast, it has been recently proposed that the structure of intratumor heterogeneity is evolutionary trait as well, evolving to maximize clonal fitness at a cancerrelevant timescale in changing (or uncertain) environment and that its structure corresponds to the bet-hedging strategy $[18,19,20,21,22]$ which has been recently put into therapeutic context [23, 24]. To sum up, the genome stays the main protagonist (i.e. selection unit) in the evolution of cancer cells, nevertheless with non-genetic heterogeneity of its eventual clone being the crucial adaptive trait at cancer-relevant, instead of proximate timescale.

Phenotypic plasticity confers to cellular tissues important properties, such as the ability of cancer cells to escape targeted therapy by switching to an alternative phenotype [25, 26, 27, 28, 29, 30]. It motivates the effort to stimulate (or prevent) specific phenotype switching purposefully as a therapeutic strategy [31], which requires deep understanding of the phenotype switching causation.
As in classical evolutionary theory are random mutations, which cause phenotypic variation, independent of selective pressure, it seems probable that reversibility of phenotype switching can be underpinned exclusively by epigenetic modifications. Regarding the therapeutical perspectives, the difference between genetic and epigenetic changes is fundamental: while the genetic changes are, in principle, irreversible, the epigenetic modifications may be reversible at the therapy-relevant timescales [26, 32, 33].

To sum up, there are well-founded reasons for studying phenotypic switching using quasispecies or population dynamics models, which address the specific characteristics of cancer. In Section 2 we present the evolutionary based framework which assumes environmental dynamics shaping the evolution of reversible switching strategies. In the quasispecies framework (see Subsections 2.1/2.3. Appendix A.1 the focus is placed on the phenotypic plasticity and reversibility. Studies of quasispecies models yielded important modelisation ideas about how to implement the immunotherapy aspects. Most of our efforts in this area have focused on the construction of the populationbased cancer models where evolution of phenotypic reversibility is incorporated together with immunotherapy. The resulting model of anticancer drug resistance are discussed in Section 3 . In Subsection 3.1 we reassess the problem of intratumoral heterogeneity and related entropy measures. The corresponding simulation results are provided in Subsection 3.2. The results respond to specific questions concerning the evolutionary nature of the drug resistance. The threshold concept has emerged as natural outcome of the simulations. Its importance was underlined by providing a more detailed supplementary analysis in the Appendix A.2

\section{Quasispecies model of pheno- typic changes}

In this section we introduce the qualitative timecontinuous evolutionary model based on the system of ordinary differential equations. The model is applied to nonequilibrium scenarios, where constrained populations of irreversible phenotypes are evolutionary drawn towards an attractor populated by reversible phenotypes.

Recently, mathematical modeling of phenotypic evolution in variable environment has received significant attention [34, 20, 35, 36], which implicitly addresses the issue of eventual adaptivity of epi- 
genetic modifications [37]. In particular, the formulation based on the quasispecies model [38] has been developed to clarify the role of mutations in the evolutionary process [39, 40, 41, 42]. Owing to its versatility, the model has found applications in a broad range of scales - from molecular and virus scales [43] up to the cellular systems, such as the populations of heterogeneous cancer cells [44, 45]. Investigation of heterogeneous malignant tissues within the context of variable environments [46] including local anticancer drug-induced environments [47] emerges as an interesting research area. The entropic variable constraints imposed on the heterogeneity were studied in [48]. In [49], the elimination of heterogeneity in the system of replicating entities was conceived as an inverse problem, with an eventual potential in therapeutical applications.

Before going into details, we highlight three salient features of the model: (i) periodicity of the (micro)environmental variations; (ii) substantial genetic diversity underlying the switching rates between isogenic phenotypes (phenotypic states); (iii) competition between the clones adopting either reversible or irreversible phenotype switching under the constraint of constant total cell population size.

Below we study effects of the variation of evolutionary rate on the fractions $c^{(z)}(k, t), k=0,1, \ldots$, $n_{s}-1$, of $n_{s}$ clones (abstract "genotypes"), each of them allowed to occupy one of the two phenotypic states, indexed by $z \in\{0,1\}$. We assume that the $k$-th genotype responds to the environmental variation in two alternative ways depending on its respective phenotypic state, $[k ; z=0]$ or $[k ; z=1]$. The time-variability of environment can be indirectly represented by the time-variability of the reproduction rates of the respective phenotypic states, $r^{(z)}(t), z \in\{0,1\}$, which are supposed to be of harmonic periodic form

$$
r^{(z)}(t)=r_{B}+(-1)^{z} \Delta r \cos (2 \pi t / T)
$$

defined by the period $T ; \Delta r$ corresponds to the degree of diversification of the reproduction fitness and $r_{B}$ to its basal level. It is worthy of noting that in [50] the oscillatory external (temperature) conditions are used to drive the evolution of the class of interacting information-carrying molecular replicators with the capability of reversible intermodal switches.

Presuming that the replication dynamics is restricted to the concentration plane $c^{(0)}(k, t) \simeq$ $c^{(1)}(k, t)$, the parameter $r_{B} \simeq\left[r^{(0)}(t)+r^{(1)}(t)\right] / 2$ may be interpreted as static effective replication rate. Many natural cycles may imply changes in the replication rate, which can be, within the context of cancer research, exemplified by the cyclic hypoxia-reoxygenation exposure within solid tumors [51].

To study quasispecies dynamics with purposefully specified the reversibility between the phenotypes $z=0$ and $z=1$ in the respective clones, we define the parameter $\varphi \in\langle 0,1\rangle$, via the linear relation

$$
\varphi\left(k, n_{s}\right)=\frac{n_{s}-k-1}{n_{s}-2}, \quad k=1, \ldots, n_{s}-1,
$$

which uniformly discretizes commitment of the cell to the respective phenotype. The labeling of the clones by the index $k$ is introduced to make next formalization more feasible. Among the possible states we highlight the neutral (or "symmetric") case $\varphi\left(n_{s} / 2, n_{s}\right)=1 / 2$ (equal commitment to either phenotype), as well as the "boundary" cases $\varphi\left(1, n_{s}\right)=1$ and $\varphi\left(n_{s}-1, n_{s}\right)=0$ corresponding to exclusive commitment to the respective phenotype, which implies complete irreversibility. In the following, the range of indices $k$ is completed with $k=0$ which represents the wild clone (neither mutations nor transitions between phenotypes are applied). Let $\left(1-\varphi\left(k, n_{s}\right)\right) c^{(1)}(k, t)$ and $\varphi\left(k, n_{s}\right) c^{(0)}(k, t)$ specify intensity of the transitions from the phenotype 0 to the phenotype 1 , and vice versa. The above terms can be combined to describe the switching flow

$$
\begin{aligned}
J_{s w}\left(k, n_{s}, t\right) & =\left(1-\varphi\left(k, n_{s}\right)\right) c^{(1)}(k, t) \\
& -\varphi\left(k, n_{s}\right) c^{(0)}(k, t),
\end{aligned}
$$

which, when incorporated into dynamics, changes phenotypic fractions corresponding to $z=0$ and $z=1$ in each of the respective clones $k=$ $1,2, \ldots, n_{s}-1$. In further, the direction of the switching flow $(-1)^{z} J_{s w}$ is controlled by the prefactor $(-1)^{z}$. Assuming non-negativity of the fractions, irreversibility of the "boundary" species is consistent with unidirectionality of the "boundary" flows $J_{s w}\left(1, n_{s}, t\right)=-c^{(0)}(1, t) \leq 0$ and $J_{s w}\left(n_{s}-\right.$ $\left.1, n_{s}, t\right)=c^{(1)}\left(n_{s}-1, t\right) \geq 0$. This sharply contrasts with the central species $k_{\text {central }}=n_{s} / 2$, where $\varphi\left(k_{\text {central }}, n_{s}\right)=1 / 2$ provides $J_{s w}\left(n_{s} / 2, t\right)=$ $(1 / 2)\left[c^{(1)}\left(n_{s} / 2, t\right)-c^{(0)}\left(n_{s} / 2, t\right)\right]$ which reflects the fact that both directions of the flow (i.e. $J_{s w} \geq 0$, $\left.J_{s w} \leq 0\right)$ are allowed for $c^{(z)}\left(n_{s} / 2, t\right)$. As $J_{s w}\left(k, n_{s}, t\right)$ is constructed without considering explicit causal sensoric response [52], it can be viewed as a population-level consequence of evolved bethedging strategy within the context of quasispecies ODE (ordinary differential equations). Note that the structure of switching term on the population level is similar to that used to describe the dichotomous switching of tumor cells [53]. Specific terms 
of this type related to the transitions between bacterial subpopulations were applied as well [54].

We postulate that the initial population is formed exclusively by the zero-th clone, which is gradually redistributed (by mutation mechanisms) among the concurrent clones, $k=1,2 \ldots n_{s}-1$. If the phenotype switching absents for $k=0$, the dynamics of the population, $c^{(z)}(0, t)$, follows

$$
\begin{aligned}
\frac{d c^{(z)}(0, t)}{d t} & =\left[r^{(z)}(t)-\Phi(t)\right. \\
& \left.-\left(n_{s}-1\right) \mu\right] c^{(z)}(0, t)
\end{aligned}
$$

and the population fraction $k=0$ changes exclusively due to irreversible mutations. Their impact is modeled by $-\left(n_{s}-1\right) \mu c^{(z)}(0, t)$ terms proportional to the positive coefficient $\mu$. The additional assumption is, that all mutants $k=1,2, \ldots, n_{s}-1$ are produced with the same rate $\mu c^{(z)}(0, t)$. The competition controls proliferation via the scalar $\Phi(t)$ term which is interpreted later. The Eq. (2.4) is considered together with the ODE system

$$
\begin{aligned}
\frac{d c^{(z)}(k, t)}{d t} & =\left[r^{(z)}(t)-\Phi(t)\right] c^{(z)}(k, t) \\
& +\mu c^{(z)}(0, t)+m(-1)^{z} J_{s w}(k, t)
\end{aligned}
$$

written for $k=1,2, \ldots, n_{s}-1$, constructed to describe combined effect of the replication, selection and switching.

The newly introduced parameter $m$ controls the intensity of the phenotype switching and can be interpreted as the measure of reversibility. The limit conditions under which the effect of $m J_{s w}$ is significantly weakened can be viewed as conditions supporting conservative bet-hedging [21]. For the sake of simplicity as well as better understanding of the model implications, the parameter $m$ is chosen to be uniform over the clones.

Regarding the structure, description and interpretation of the switching process at the level of ODE, we note that: (i) it follows from the Eq.(2.5) that the total rate of the clonal fractions $d\left(c^{(0)}(k, t)+c^{(1)}(k, t)\right) / d t$ loses explicit dependence on $J_{s w}(k, t)$; (ii) without mutation and replication terms the particular phenotypic equilibria can be formed when $\left[J_{s w}(k)\right]_{e q u i l} \stackrel{!}{=} 0$, which leads to the ratio $\left[c^{(1)}(k) / c^{(0)}(k)\right]_{\text {equil }}=\varphi\left(k, n_{s}\right) /\left[1-\varphi\left(k, n_{s}\right)\right]$. The competition among the clones is described conveniently using constraint of constant overall population density

$$
c_{\Sigma}^{(0)}(t)+c_{\Sigma}^{(1)}(t)=1
$$

with the particular terms

$$
c_{\Sigma}^{(z)}(t)=\sum_{k=0}^{n_{s}-1} c^{(z)}(k, t), \quad z=0,1 .
$$

If the sum of the left and right hand sides of Eq.(2.4) and Eq.(2.5) is carried out, and the result is compared with Eq.(2.6), the scalar correction to the reproduction rate may be constructed as

$$
\Phi(t)=\sum_{z=0,1} r^{(z)}(t) c_{\Sigma}^{(z)}(t) .
$$

In order to reduce the initial asymmetry, we have used in our simulations (see Subsection 2.3 in below) of quasispecies model the initial conditions

$$
c^{(z)}(k, t=0)=\frac{\delta_{k, 0}}{2}, \quad z \in\{0,1\},
$$

where $\delta_{k, 0}$ is the Kronecker delta. It means, that in the case of the initial demise of the $k=0$ species, we expect the repopulation towards the species $k=1,2, \ldots, n_{s}-1$. Since the mutations contribute uniformly to the expansion of all remaining clones $(k \neq 0)$ with the same rate $\mu c^{(z)}(0, t)$, the decisive contribution to the disparity of the fractions is expected solely from the switching mechanism where diversification is guaranteed by the use of $\varphi\left(k, n_{s}\right)$.

\subsection{Entropy measures of heterogeneity}

The general concept of entropy is used in various scientific fields and can therefore be perceived differently according to the purposes of the information processing in the respective areas of biological research. Shannon entropy is an example of the key systemic measure designed to quantify the information storage, transfer or system heterogeneity/diversity. One key step in this direction is the idea to join a fitness concept to informationbased characteristics by the value of information [55]. According to [56], the entropy-based measure can be used as a predictive indicator of the evolutionary efficiency in avoiding extinction. The measures can quantify diversity within the context of phenotypic switching [57] analogously as in presented work. In the field of cancer biology and diagnostics, biostatistics, as well as in the studies of cellular composition, the entropy can be utilized to monitor and characterize dynamics of heterogeneity at many levels. Shannon entropy is currently considered the most promising robust metrics and the tumor-specific imaging biomarker to enable rational prognostic analysis based on the data gathered from CT-scans [58]. 
In our specific context, to characterize selection strength and asymptotic behavior at the systemic level, we focus on the measures of heterogeneity of evolving population fractions. To quantify the dynamic heterogeneity we utilize two different measures. The first of them is Shannon entropy

$$
S_{E P I G}(t)=-\sum_{z=0,1} \sum_{k=0}^{n_{s}-1} c^{(z)}(k, t) \ln c^{(z)}(k, t),
$$

which reflects the epigenetic information. It is obvious, that this form is sensitive to the arrangement of phenotypic fractions. On the contrary, the introduction of measures based on the total concentra- tion

$$
c(k, t)=c^{(0)}(k, t)+c^{(1)}(k, t)
$$

or another meaningful algebraic combinations of $c^{(0)}, c^{(1)}$ is a way how to abandon phenotypic details (including subtle oscillations). Therefore, the genetic heterogeneity and variability can be better described by

$$
S_{G}(t)=-\sum_{k=0}^{n_{s}-1} c(k, t) \ln (c(k, t))
$$

Both $S_{E P I G}$ and $S_{G}$ measures can be constructed regarding often used analogy between probability and occupancy fraction [49].

(A)
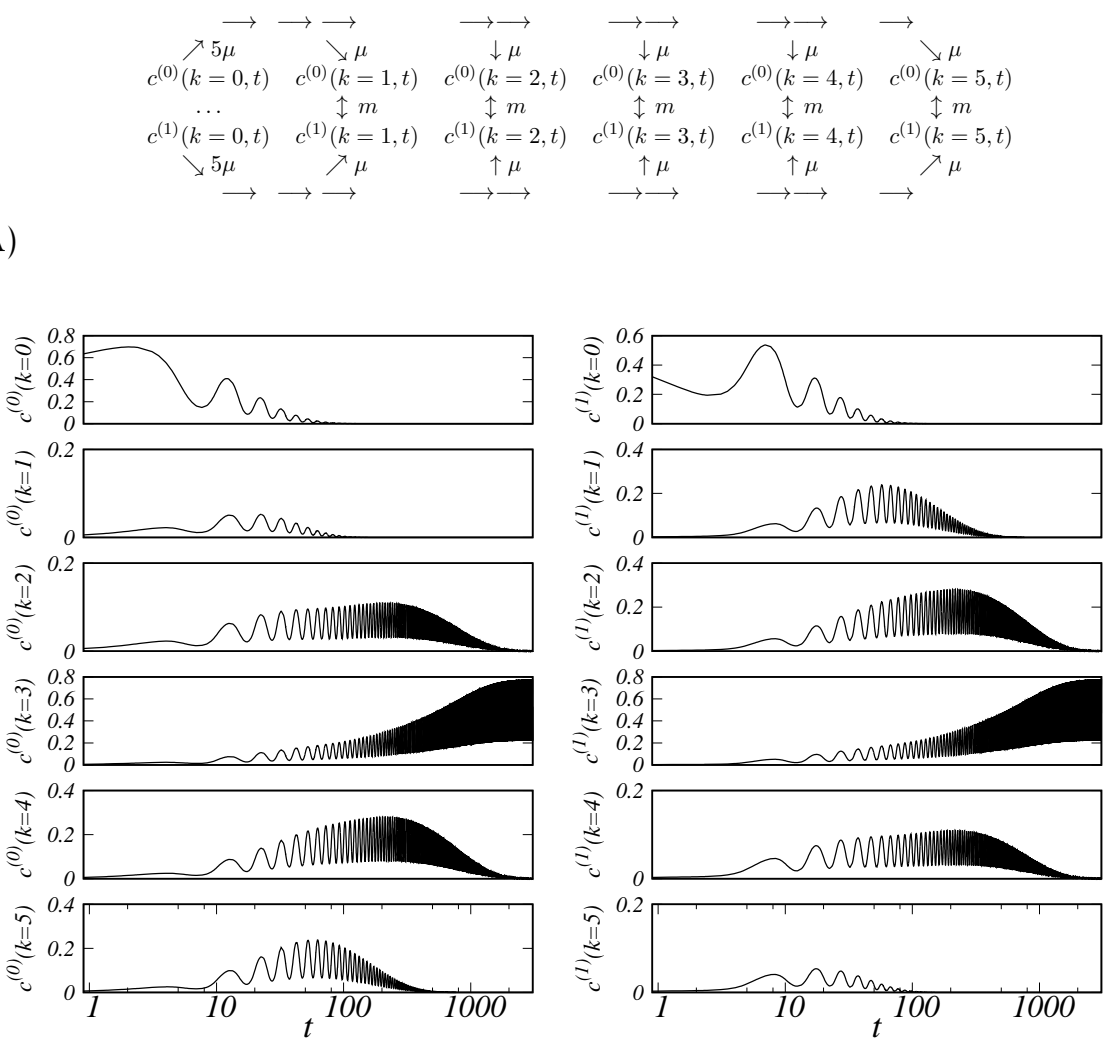

(B)

Figure 2.1: Panel (A): Schematic diagram of the quasispecies model $\left(n_{s}=6\right)$ introduced in Section 2, Special emphasis is placed on the reversibility imposed by $\varphi$. Panel (B): The time dependence of $n_{s}=6$ species fractions in periodic environment $(T=10)$. The calculations have been performed for the parameters $\Delta r=0.4$, $\mu=0.01, m=0.05$. The highest (effective) replication rate observed for the highest reversibility corresponding to $\varphi\left(3, n_{s}\right)=1 / 2$. The fractions of the other species are suppressed in the long run (limit cycle regime).

\subsection{Effective replication rate of the clone}

To investigate the behavior of the system of equations (2.4) and 2.5 we proposed an alternative effective description. Within this, the effective dy- namics of total fraction $c(k, t)$ (see Eq.(2.11) can be expressed by simple formula

$$
\frac{d c(k, t)}{d t}=\left(r_{e f f}(k, t)-\Phi(t)\right) c(k, t) .
$$




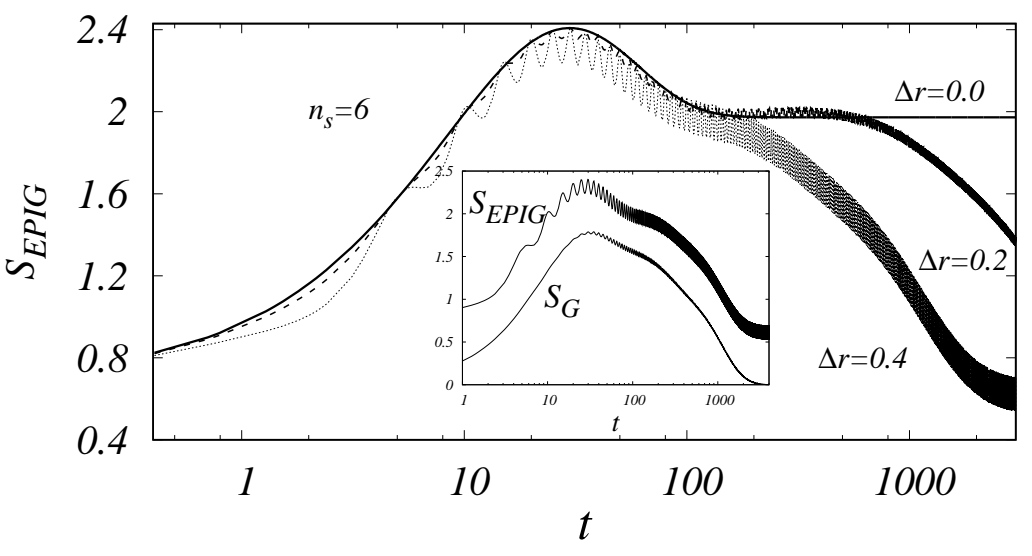

(A)
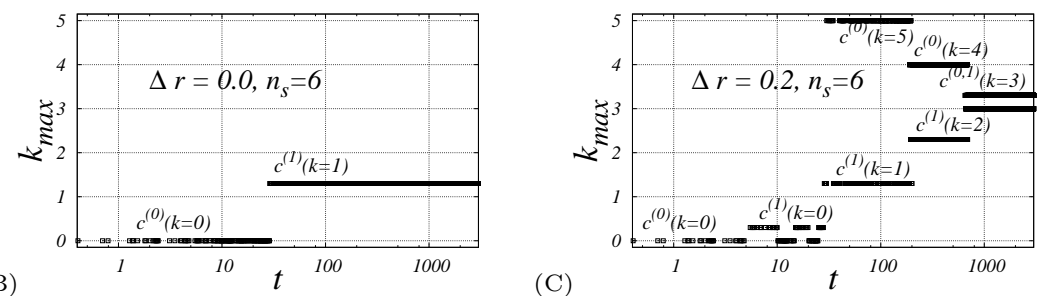
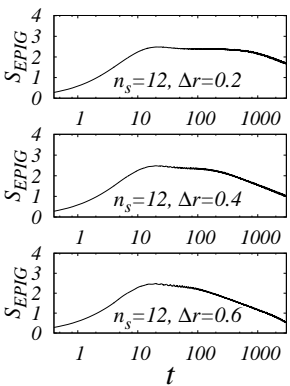

(D)

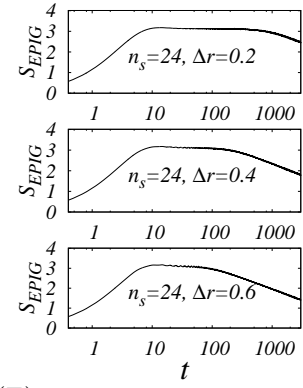

(E)

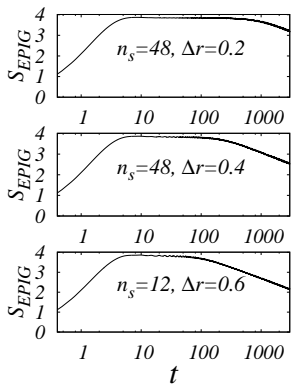

$(\mathrm{F})$

Figure 2.2: Panel (A) shows the time dependence of the entropy measure $S_{E P I G}$ (the inset includes comparison with $\left.S_{G}\right)$. The difference between the sub-threshold $(\Delta r=0$, absence of the variation) and superthreshold fitness environmental variations (alternatives $\Delta r=0.2,0.4$ ). Calculated for the period $T=10$. Remaining undetermined parameters are $\mu=0.01$ and $m=0.05$. In the panels (B) and (C) we plot the time dependencies of the effective index $k_{\max }(t)+z_{\max }(t) \times 0.3$. Here symbol max is related to the species of the maximal abundance and the auxiliary constant 0.3 is chosen for the visualization purposes only. For example in the case $z_{\max }=0$ the plot hits the mesh line [see $c^{(0)}(5), c^{(0)}(4), c^{(0)}(3), c^{(0)}(0)$ in the panel $(\mathrm{C})$ ] corresponding to the species of the maximal instant concentration showing the enhanced selection for the phenotype with highest reversibility, i.e. for $\varphi=1 / 2, \varphi /(1-\varphi)=1$. In panel (B), the evolution in the static environment remains frozen at $k_{\max }=1, z_{\max }=1$, whereas the evolution obtained for $\Delta r=0.2$ in panel (C) asymptotically supports the selection for the phenotype with highest degree of reversibility $k_{\max }=3, z_{\max }=0,1$ and $\varphi(3)=1 / 2$. (The results obtained for higher $\Delta r$ are qualitatively similar.) Panels (D), (E), (F) depict effects of the number of clones $n_{s} \in\{12,24,48\}$ and $\Delta r \in\{0.2,0.4,0.6\}$ on the $S_{E P I G}(t)$.

Here, the effective replication rate of the $k$-th clone $r_{e f f}(k, t)$ plays a key role for many results to follow. The phenomenology avoids the formal use of mutations, switching or $c^{(0)}(k, t), c^{(1)}(k, t)$. The summation of the equations Eq. (2.13) leads to the formula

$$
\Phi(t)=\sum_{k=0}^{n_{s}-1} r_{e f f}(k, t),
$$

which implies the interpretation of $\Phi$ as a total replication rate. Moreover, the consistence of Eq.(2.14) and Eq. (2.8) can be accomplished for the "mixed-type" solution (see also [36])

$$
r_{e f f}(k, t)=r^{(0)}(t) c^{(0)}(k, t)+r^{(1)}(t) c^{(1)}(k, t) .
$$




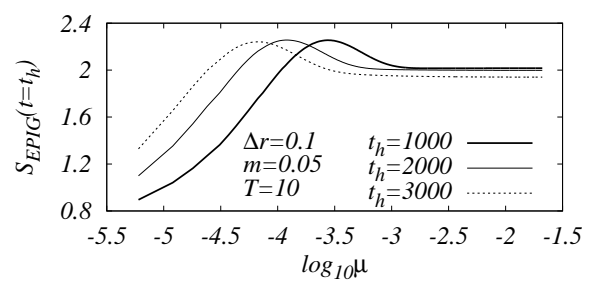

(A)
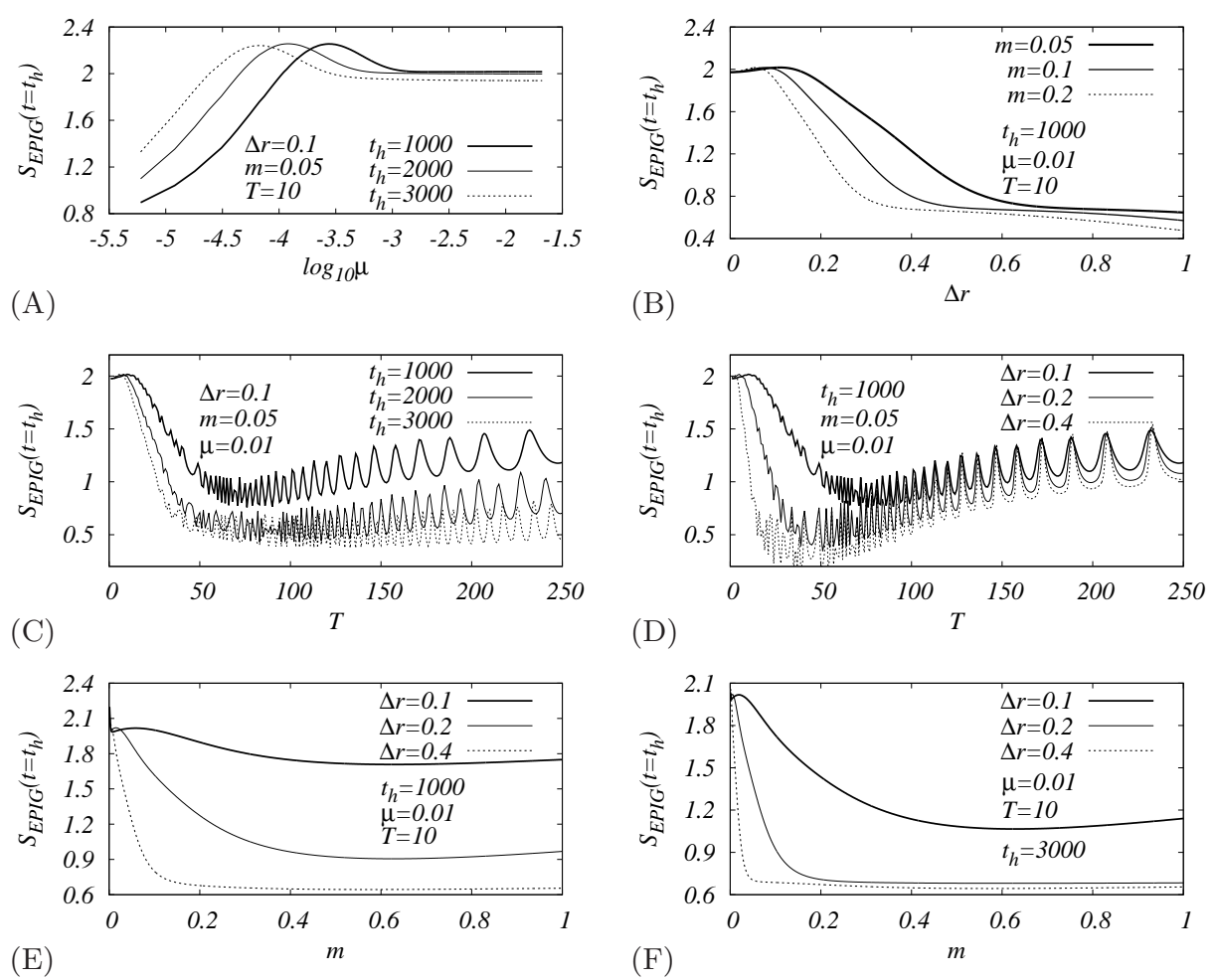

Figure 2.3: The differences in the efficiency of selecting extreme reversibility within the frame of nonequilibrium evolutionary dynamics. The panels show dependencies of the entropy measure $S_{E P I G}\left(t=t_{h}\right)$ calculated for various combinations of parameters and time horizons $t_{h} \in\{1000,2000,3000\}$. Panel (A) involves the threshold dependencies on the mutation rate $\mu$. Note that sufficiently large $\mu$ is needed to overcome the initial barrier of forming heterogeneous state from which selection can be realized. According to panel (B) the more intense environmental changes, the faster selection for the most reversible clone. Panels (C), (D) indicate non-monotonous dependence on $T$. In case (D), obtained for $\Delta r \in\{0.1,0.2,0.4\}$, the efficiency of selection reduces for the wide range of $T$ until global minima $T \sim(25-90)$ are attained. Obviously, as shown in panels $(E),(F)$, selection mechanism can be easily accelerated by increasing the switching rate parameter $m$.

\subsection{Numerical investigation}

Apart from a few numerical exceptions, listed explicitly in the figure captions, the simulations have been performed for $n_{s}=6$ species and the parameters $T=10, r_{B}=1, \Delta r \in\{0.1,0.2,0.4\}, m=0.05$, $\mu=0.01$. In agreement with intuitively expected behavior of the model, Fig. 2.1 shows gradual qualitative progress in the redistribution of the species fractions. At the intermediate time scales (10a.u.100 a.u.), there is a period of "hesitancy", where all the clones $k>1$ nearly counterbalance their respective competitors. During this short initial phase, the variable environment causes that the putative equality among the clones $k=1,2, \ldots, 6$ gradually vanishes and the highest reversibility due to $\varphi=1 / 2$ corresponding to the ratio $\varphi /(1-\varphi)=1$ attributed to the clone $k_{\text {central }}=n_{s} / 2=3$, leads at the long run $(\geq 1000)$ to the largest $r_{\text {eff }}$ [see the analytical approximation given by Eq. A.10 in the Appendix A.1.

Fig 2.2 shows the non-trivial transient states and the initial increase common for $S_{E P I G}$ and $S_{G}$. The largest differences between $S_{E P I G}$ and $S_{G}$ are localized mainly in the long-time asymptotics. Here, the main question arises to what extent environmental variability affects the long-term heterogeneity of the populations. There are many indicia that populations obtained for large and small $\Delta r$ significantly differ. Fig 2.2 depicts both the entropy measures as functions of $\Delta r$, which is the main determinant of environmental variability. The variability causes the convergence of $S_{E P I G}$ to the positive limit value, while trivial limit $S_{G} \rightarrow 0$ indicates dominance of the single species. For small $\Delta r$, there are only very low evolutionary benefits due to switching between phenotypic states, which leads to extremely slow evolution. This 
means that numerical approach becomes inappropriate to capture the subtle differences between non-equilibrium decay and long-time limit cycle behavior.

The numerical problems stimulated development of analytical approximation discussed in Appendix A.1 Both approaches are consistent in indicating of the fact that convergence to the most reversible clone is faster as $\Delta r$ increases [see panel (C) of Fig[2.2]. In addition, it appears that for given time horizon $t_{h}$ the increase in $\Delta r$ leads to the threshold-like behavior [see Fig 2.3 (B)]. According to Fig 2.2(B), without sufficient environmental variability most of the population remains trapped in the clone $k=1$. Fig 2.3 summarizes the entropic responses induced by $\mu, \Delta r, T$ and $m$. For example, panels (C) and (D) show how the choice of $T$ affects the efficiency of the selection of the most reversible strategy. It turns out that large enough $T$ supports rapid selection. In agreement with the intuitive expectations, the process can be reinforced by super-threshold value of $m$.

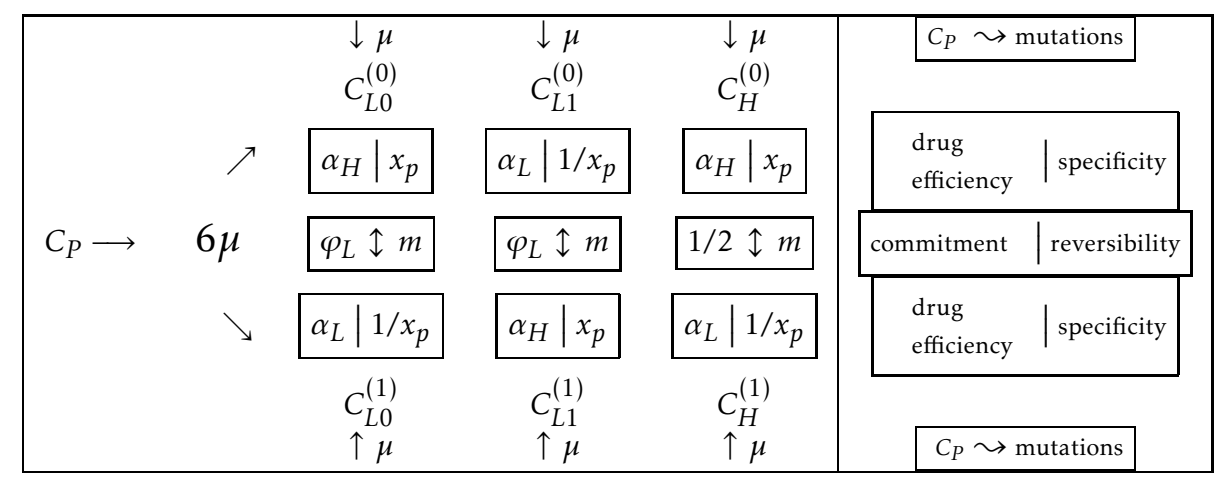

Table 2.1: The scheme depicts the relations between abundances $C_{P}, C_{L 0}^{(z)}, C_{L 1}^{(z)}, C_{H}^{(z)} ; z \in\{0,1\}$ mutation $(\sim \mu)$ and switching $(\sim m)$ processes; the variants of phenotypic state commitments $\left\{\varphi_{L}, 1 / 2\right\}$ [see Eq.(3.5)], drug efficiencies $\left\{\alpha_{L}, \alpha_{H}, 1\right\}$ [see Eq.(3.7)], and drug specificities $\left\{x_{p}, 1 / x_{p}\right\}$ [see Eq.(3.8)] within the model represented by Eq.(3.9).

\section{Model of immunotherapy}

In the next we apply the above framework to investigate evolutionary aspects of the resistance to immunotherapeutic drugs caused by phenotypic reversibility. We built the new model using the exogenous simplified variant of the Kirschner-Panetta (KP) model of immunotherapy [59]. The original KP model consists of the three autonomous equations, for the effector cells production, tumor growth with tumor clearance, and, finally, equation for cytokine interleukin-2 (IL-2) production. Essential in the original $\mathrm{KP}$ model is the boosting of the IL-2 against tumors. There are many versions of the model that include simplified but biologically plausible approaches [60]. In [61] the gene therapy characterized by populations of effector and cancer cells was described by means of two coupled autonomous ODE. Recently, twopopulations cancer model [62] with competitive interaction has been used to investigate the combination of immunotherapy and chemotherapy.

Here, we present a simplified model of a single cancer clone where a population of cancer cells varies accordingly to exogenous ODE

$$
\frac{d C}{d t}=r C(1-C)-a \frac{e(t) C}{C+g} .
$$

Consistently with the original KP model, the efficiency of therapeutic interventions is described by the clearance parameter $a$. The parameter $g$ plays the role analogous to that in the MichaelisMenten kinetics. Regarding the treatment classification based on the rate of tumor cells killing, the model term $-a e(t) C /(C+g)$ corresponds to the cytotoxic activity of non-logkill type [63]. Unlike the quasispecies model, the rate of replication $r$ does not change. The classical logistic term $r C(1-C)$ is used for the nutrient-limited cancer growth of the rescaled abundance of tumoral cells $C$. Its use [loosely analogous to $\Phi$ from Eq.(2.14)] causes that tumor size stabilizes at $C \leq 1$. (The carrying capacity is equal to 1 in here adopted scaling).

Dynamics of environment is mediated by the population of the effector cells. We assume that population size $e(t)$ varies according formula

$$
e(t)=e_{B}+\Delta e \cos \left(\frac{2 \pi t}{T}\right) .
$$


This simplification exploits a few aspects: (i) the exogenous formulation is parametrically less demanding than the original autonomous (endogenous) KP model; (ii) it can be easily linked to the harmonic model of replication rate $r^{(z)}(t)$ proposed in Eq.(2.1) with the exception that it cancels the dependence on $z \in\{0,1\}$ (the phenotypic state dependence is restored in the Subsection 3.1 by introducing additional parameters to represent therapeutic interventions); (iii) it avoids the ODE stiffness problems typical of the original KP formulation; (iv) the harmonic character of Eq.(3.2) is qualitatively consistent with KP phase diagrams [59] belonging to the dynamical regimes with some limit cycle attractors; (v) periodicity is consistent with the therapeutic option for immuno-oncological dynamics [64].

Before studying more complex numerical examples, we continue with the parametrization of the elementary model based on Eq.(3.1) and Eq. (3.2). According to the results, the system preliminary simulated with the initial condition $C(0)=0.1$ for ten periods exhibits the largest susceptibility to the changes in $g$ for the parameters

$$
\begin{array}{lll}
e_{B}=0.5, & \Delta e=0.4 & \text { (analogs of } \left.r_{B} \text { and } \Delta r\right), \\
g=0.45, & a=1, & T=10, \quad r=1 \text { (fixed). }
\end{array}
$$

The parameters are intended for further simulations of multiclonal populations of cancer cells.

\subsection{Diversification of the effects of im- munotherapy}

To illustrate the role of reversibility of phenotypic switching in the evolution of the therapy resistance, we construct the evolutionary model where reversibility is considered along with intratumor heterogeneity reflected by the clonedependent and state-specific sensitivity to drug. It works with the population of seven rescaled instant species abundances, forming the set $\operatorname{set}_{E}(t) \equiv$ $\left\{C_{P}(t), C_{L 0}^{(0)}(t), C_{L 0}^{(1)}(t), C_{L 1}^{(0)}(t), C_{L 1}^{(1)}(t), C_{H}^{(0)}(t), C_{H}^{(1)}(t)\right\}$, where $C_{P}(t)$ denotes the abundance of the primary cancer cells.

Four abundances $C_{L 0}^{(z)}(t), C_{L 1}^{(z)}, z \in\{0,1\}$ correspond to two clones $L 0, L 1$ with low reversibility (indexed as $L$ ) between states. The clones are characterized by the commitment $\varphi_{L} \in(0,1 / 2)$. Here, the a priori tendency toward low reversibility (with the obvious exception for $\varphi_{L}=1 / 2$ ) is ensured by the combination of "asymmetric" commitments $\varphi_{L}, 1-\varphi_{L}$ (which occur in the complementary pairs of the switching flows). The supplementary lower numerical index 0 in $C_{L 0}^{(z)}$ (or 1, respectively) indicates that immunotherapy is specially designed to have more profound impact on the phenotypic state 0 (or 1) (therapeutic effectiveness is below denoted as $\left.\alpha_{H}\right)$, whereas the alternate phenotypic state is eliminated with lower efficiency $\left(\alpha_{L}\right.$, see below). The commitment equal to $1 / 2$ (high reversibility) is imposed on the clone $H$ characterized by the abundances $C_{H}^{(0)}, C_{H}^{(1)}$.

The overview of the theoretical structure of the model is schematically highlighted in Table 2.1. In contrast to the quasispecies model, the sum

$$
C_{\Sigma} \equiv C_{P}+\sum_{z=0,1}\left(C_{L 0}^{(z)}+C_{L 1}^{(z)}+C_{H}^{(z)}\right)
$$

is not normalized to unity but, instead, $\left(1-C_{\Sigma}\right)$ is used to construct the logistic form $r C\left(1-C_{\Sigma}\right)$ which guarantees $C_{\Sigma}(t) \leq 1$ for proper initial conditions. Similar term that sets upper limit on the abundance of the mixed populations can be found elsewhere [65, 66, 62].

As we pay attention to the problem of reversibility within the context of heterogeneity, the switching flows within the clones $L 0, L 1$, and $H$ are defined as it follows

$$
\begin{aligned}
J_{s w L 0} & =\left(1-\varphi_{L}\right) C_{L 0}^{(1)}-\varphi_{L} C_{L 0}^{(0)}, \\
J_{s w L 1} & =\left(1-\varphi_{L}\right) C_{L 1}^{(1)}-\varphi_{L} C_{L 1}^{(0)}, \\
J_{s w H} & =\frac{1}{2}\left(C_{H}^{(1)}-C_{H}^{(0)}\right) .
\end{aligned}
$$

All population rates are defined by the universal formula consisting of the adaptations of Eq. (3.1) and former variant of the switching flow model

$$
\begin{aligned}
& \mathcal{R}\left(\tilde{C}, \tilde{J}, \tilde{\alpha}, N_{\mu}\right)=r \tilde{C}\left(1-C_{\Sigma}\right) \\
& \quad+m \tilde{J}-\tilde{\alpha} a \frac{e(t) \tilde{C}}{C_{\Sigma}+g}+N_{\mu} \mu C_{P},
\end{aligned}
$$

where $\tilde{C}, \tilde{J}, \quad \tilde{\alpha}, N_{\mu}$ are some auxiliary general variables which are substituted by some specific variables and constants: $\tilde{C} \in \operatorname{set}_{E}, \tilde{J} \in$ $\left\{J_{s w L 0}, J_{s w L 1}, J_{s w H}\right\}, \tilde{\alpha}=\left\{\alpha_{L}, \alpha_{H}\right\}, N_{\mu} \in\{-6,+1\}$. In comparison with Eq.(3.1), we also include mutation mechanism $\left(\sim N_{\mu} \mu C_{P}\right)$ and switching $(\sim m \tilde{J})$ already used in the quasispecies simulations.

The contributions related to the switching process are adopted from [53]. The alternatives $J_{s w L 0}$, $J_{s w L 1}, J_{s w H}$ (might loosely correspond to "escape routes" for the respective cancer sub-populations [25]. Conversions between drug-resistant and drug-sensitive clones are the main issue of the resulting construction considered in line with the abstract model [67] used as a prototype. To study bacterial persistence under conditions of antibiotic stress, a similar, two-component population 
model was proposed in [68]. Finally, the technical details of simultaneous incorporation of mutations and the switching processes into the model of epigenetically structured population can be found elsewhere [69].

To introduce the clone- and state-dependent sensitivity to drug into the model, the scalar clearance parameter $a$ in Eq. (3.1) is in Eq. (3.6) replaced by the species-dependent term $\alpha_{j}^{(z)} a, j \in\{0,1\}, z \in$ $\{0,1\}$ with the prefactor representing a modifier of the drug efficiency of the phenotype $z$ of the clone $j$. To stay consistent with the formal structure of the model, $\alpha_{j}^{(z)}$ is represented by four matrix elements, $\alpha_{0}^{(0)}, \alpha_{0}^{(1)}, \alpha_{1}^{(0)}, \alpha_{1}^{(1)}$, related to the particular clone and phenotype. Nevertheless, to keep the conceptual model instructive, we assume only two basic levels in the matrix elements, one for diagonal, one for non-diagonal elements, respectively

$$
\left(\begin{array}{cc}
\alpha_{0}^{(0)} & \alpha_{1}^{(0)} \\
\alpha_{0}^{(1)} & \alpha_{1}^{(1)}
\end{array}\right)=\left(\begin{array}{cc}
\alpha_{H} & \alpha_{L} \\
\alpha_{L} & \alpha_{H}
\end{array}\right) .
$$

We note that diversity of the forms of $\mathcal{R}$ conditioning diversity within $\operatorname{set}_{E}$ stays sufficient as it is not based explicitly on the effect of $\tilde{\alpha} \in\left\{\alpha_{L}, \alpha_{H}\right\}$, but on the possible combinations of $\tilde{\alpha}$ with the commitment terms $\varphi_{L}, 1-\varphi_{L}, \frac{1}{2}$ coming from $J_{s w L 0}, J_{s w L 1}$ and $J_{H}$ as well.

Both $\alpha_{L}, \alpha_{H}$, representing low/high therapeutic effects, respectively, can be further parametrized by the single auxiliary parameter $x_{p} \geq 1$

$$
\alpha_{H}\left(x_{p}\right)=x_{p}, \quad \alpha_{L}\left(x_{p}\right)=\frac{1}{x_{p}} .
$$

The parametrization is designed to satisfy requirements $\alpha_{0}^{(z)} \alpha_{1}^{(z)}=\alpha_{L}\left(x_{p}\right) \alpha_{H}\left(x_{p}\right)=1$ and $0<\alpha_{L} \leq$ $1 \leq \alpha_{H}$ for both phenotypic states $z \in\{0,1\}$. In the special boundary case $x_{p}=1$ which implies $\alpha_{L}(1)=\alpha_{H}(1)=1$, there is no modification of $a$. To sum up, the parameter $x_{p}$ can be interpreted as a measure of specificity of immunotherapeutic intervention. To present a microscopic view of the problem studied, we outline an interpretation in which $x_{p}$ integrates the basic mechanisms, such as the specific efficacy of the tumor-associated antigens, the ability of tumors to activate T-cell responses in- cluding the tumor recognition and the induction of cancer cell deaths [70].

By combining Eq. (3.5) with Eq. (3.6), the population dynamics of $(1+6)$ tumor species can be expressed as it follows

$$
\begin{aligned}
\frac{d C_{P}}{d t} & =\mathcal{R}\left(C_{P}, 0,1,-6\right), \\
\frac{d C_{L 0}^{(z)}}{d t} & =\mathcal{R}\left(C_{L 0}^{(z)},(-1)^{z} J_{s w L 0}, \alpha_{0}^{(z)},+1\right), \\
\frac{d C_{L 1}^{(z)}}{d t} & =\mathcal{R}\left(C_{L 1}^{(z)},(-1)^{z} J_{s w L 1}, \alpha_{1}^{(z)},+1\right), \\
\frac{d C_{H}^{(z)}}{d t} & =\mathcal{R}\left(C_{H}^{(z)},(-1)^{z} J_{s w H}, \alpha_{0}^{(z)},+1\right) .
\end{aligned}
$$

The first formula of the list expresses that phenotype switching absents (zero at the second position of $\mathcal{R}$ ) in the primary tumor clone. This choice is consistent with the quasispecies model. In addition, there is no modification of the drug sensitivity $a(\tilde{\alpha}=1)$. The factor $(-6)$ expresses that mutation process modifies the primary tumor growth by subtracting rate factor $6 \mu C_{P}$. We assume that the "most reversible" and "symmetric" transitions between phenotypes corresponding to $C_{H}^{(0)}$ and $C_{H}^{(1)}$ are violated by the factor $\alpha_{0}^{(z)} \neq 1$.

As in the case of quasispecies model, now we propose measures that allow better interpretation of the results. Again, the appropriate transformations allow us to define proper measures of heterogeneity. For all $C_{E} \in \operatorname{set}_{E}$ the rescaling $\left(C_{E} / C_{\Sigma}\right)$ can be performed, that guarantees $\sum_{C_{E} \in \text { set }_{E}}\left(C_{E} / C_{\Sigma}\right)=1$. In this case, the tumor can be monitored by epigenetic entropy-like measure

$$
S_{E P I G}^{C}=-\sum_{C_{E} \in \text { set }_{E}} \frac{C_{E}}{C_{\Sigma}} \ln \frac{C_{E}}{C_{\Sigma}} .
$$

In analogy with Eq.2.11, we define four-element auxiliary set $\operatorname{set}_{G} \equiv\left\{C_{P}, C_{L 0}^{(0)}+C_{L 0}^{(1)}, C_{L 1}^{(0)}+C_{L 1}^{(1)}\right.$, $\left.C_{H}^{(0)}+C_{H}^{(1)}\right\}$ which allows modification of the original definition of Eq.(2.12) to the form

$$
S_{G}^{C}=-\sum_{C_{G} \in \operatorname{set}_{G}} \frac{C_{G}}{C_{\Sigma}} \ln \frac{C_{G}}{C_{\Sigma}} .
$$

\subsection{Simulation results - evolution of the therapeutic resistance \\ initial conditions}

The results of numerical simulations are depicted in Fig 3.1 and Fig. 3.2 They are obtained for the

$$
\begin{aligned}
C_{P}(0) & =C_{P 0} \\
C_{L 0}^{(z)}(0) & =C_{L 1}^{(z)}(0)=C_{H}^{(z)}(0)=0, \quad z \in\{0,1\}
\end{aligned}
$$



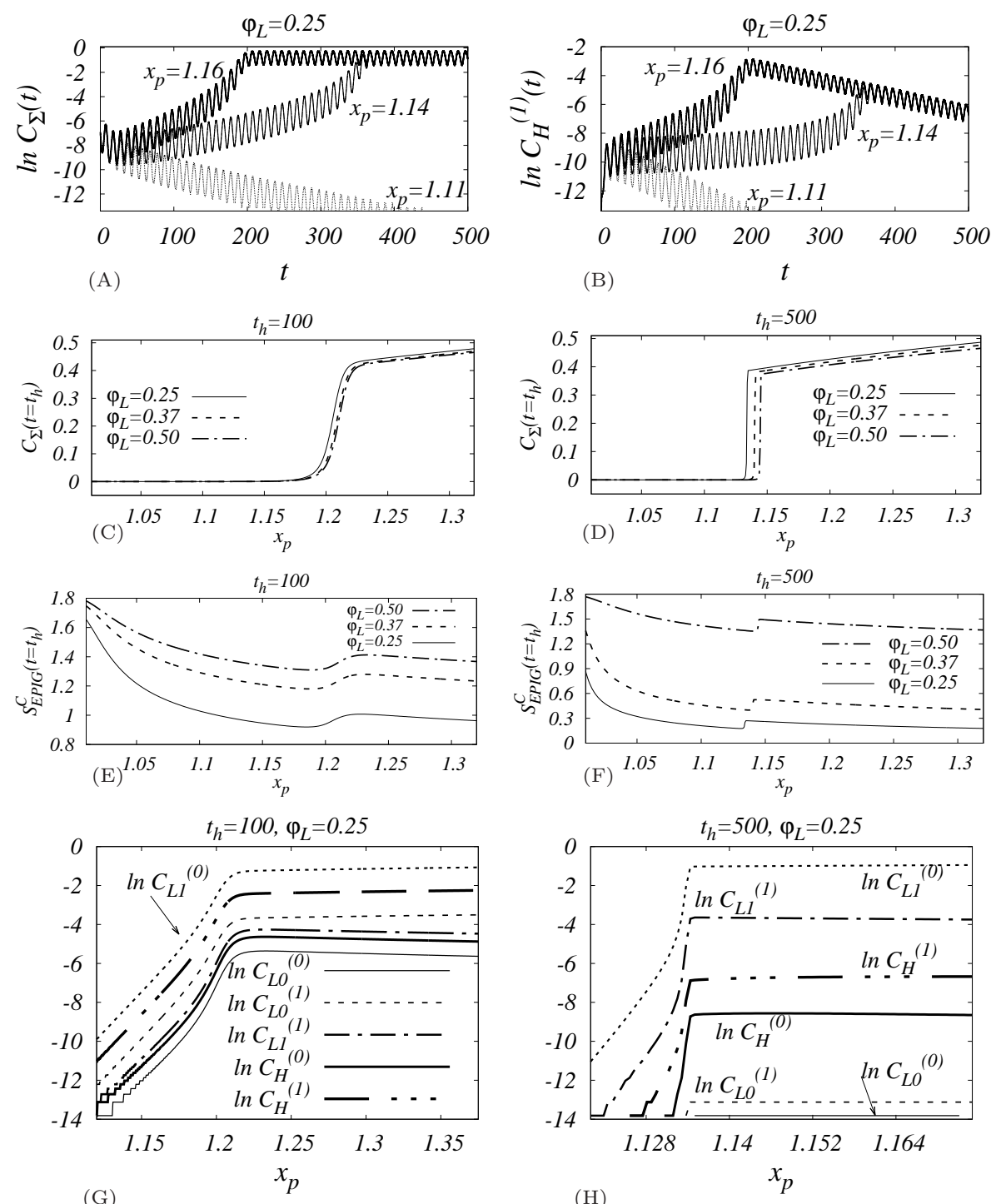

Figure 3.1: The numerical results obtained for the model incorporating evolution of reversibility and resistance to immunotherapy. In addition to other parameters we kept $\mu=0.01, m=0.05$ and $x_{p}, \varphi_{L}, t_{h}$ specified in the panels. The tumor is variable in size $\left(C_{\Sigma}(t)\right)$ and phenotypic structure $\left(C_{E} \in \operatorname{set}_{E}\right)$. Panels (A), (B) show some differences in the course of the tumor progression $\left(x_{p}=1.16\right.$, "rapid progression"; $x_{p}=1.14$, "slower" progression; $x_{p}=1.11$, "shrinking") for three selected values of $x_{p}$ characterizing qualitative differences in the therapeutic effects on the phenotypic states; (e.g. drug specificity $x_{p}=1.11$ implies $\alpha_{L} \simeq 0.9009$ ); Panel (B) shows specific phenotypic dynamics. It shows that despite the high reversibility [commitment $1 / 2$ incorporated into $J_{s w H}$ in the case of clone $\left.[H ; z \in\{0,1\}]\right]$, the abundance $C_{H}^{(1)}(t)$ drops at the late times as a result of the loss of the ability to compete. Panels (C), (D), ... (G), (H) reveal details of $x_{p}$ influence calculated for two time horizons $t_{h} \in\{100,500\}$. The threshold between slow and rapid tumor progression can be identified. The threshold character is confirmed by the fact, that the transitions due to $x_{p}$ are getting sharper as the $t_{h}$ increases. The sharpening due to a change in $t_{h}$ from $t_{h}=100$ to $t_{h}=500$ is also noticeable for the panels $(\mathrm{G})$, $(\mathrm{H})$, where more detailed information on the population structure is available. The panels $(\mathrm{E}),(\mathrm{F})$ depict the formation of the threshold values in the Shannon entropies as heterogeneity measures. Panels $(\mathrm{G}),(\mathrm{H})$ show that $C_{L 1}^{(0)}$ dominates over a wide range of $x_{p}$ although the corresponding $\varphi_{L}=0.25$ is quite far from the ideal reversibility corresponding to $1 / 2$. From panel $(\mathrm{H})$ we see that the significant long-term desirable effect of therapy persists only in the case of $C_{L 0}^{(0)}$ and partly in the case of $C_{L 0}^{(1)}$. 

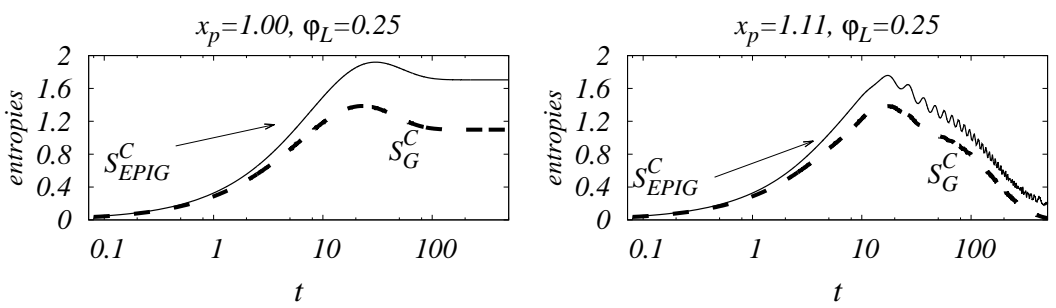

(A)
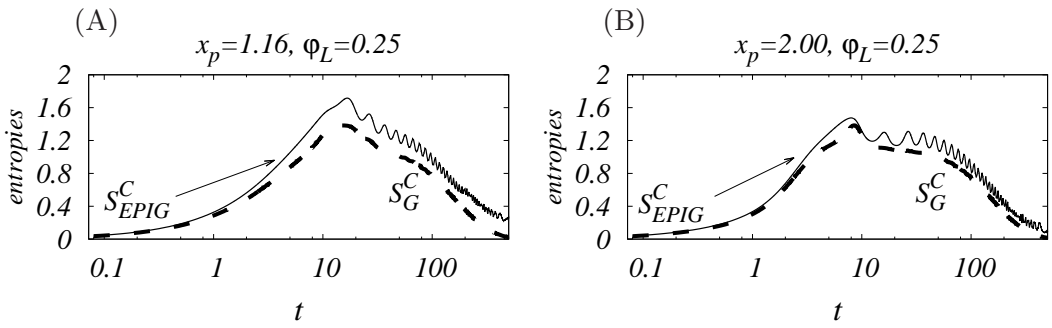

(C)

(D)

Figure 3.2: The evolutionary dynamics reflected by the heterogeneity measures $S_{G}^{C}(t)$ and $S_{E P I G}^{C}(t)$. The numerical results obtained for the additional parameters $\mu=0.01, m=0.05$ and $x_{p}, \varphi_{L}$ listed on the top of figure panels. The scenarios corresponding to different $x_{p}$ are qualitatively similar: the initial increase associated with a large number of possibilities is replaced in the peak zones by the favorable growth of selected clones, i.e. clonal expansion. A weakened preference due to $x_{p}=1$ in panel (A) leads to the highest entropy among the considered outcomes. Panels (B), (C) show the transition stages. Panel (D) indicates that differences between epigenetic and genetic entropy measures are almost entirely neutralized if selective pressures due to immunotherapy are strong enough. For (B), (C), (D) panels the after peak behavior of $S_{G}^{C}(t)$ changes smoothly contrary to "jagged" form of $S_{E P I G}^{C}(t)$.

In the following calculations the parametric value $C_{P 0}=0.001$ and parameters defined by Eq. (3.3) from the Section 3 are used, with the exception of $x_{p}$ and $\varphi_{L}$, which are listed in the respective figure captions. The applied initial conditions resemble those considered in the work [47].

As is clear from the respective panels of Fig. 3.1 the different combinations of the drug specificity parameter $x_{p}$ and phenotypic commitment parameter $\varphi_{L} \in\{0.25,0.37,0.5\}$ trigger different competitive behaviors, which are manifested for different time horizons $t_{h} \in\{100,500\}$.

A more detailed look in the panels (A), (B) of Fig 3.1 which show the monitoring of $C_{\Sigma}$ and $C_{H}^{(1)}$ reveals additional systemic features. For such characteristics, quite strong preference defined by $x_{p}=$ 1.16 implies very rapid growth, with as few as 200 time units nearly sufficient to achieve carrying capacity. Although the abundance $C_{\Sigma}$ stabilizes at its upper limit, there are still internal changes in the clonal subpopulations which are visible in variations of $C_{H}^{(1)}$. The competition under saturated conditions leads to the loss of $C_{H}^{(1)}$ dominance, giving advantage to other clones. Owing to locating simulation intentionally in the area with a rela- tively high sensitivity to $x_{p}$, even a small reduction to $x_{p}=1.14$ makes anticancer effect more apparent. In such case, 300 time units are necessary to attain saturation. This slowing down of the progression relates to the fact that more distributed therapy weakens not only dominant but, at least partially, other phenotypic variants as well. The dynamics presented in the panels (A), (B) enabled us to focus on the appropriate time horizons $t=t_{h}$ for which, consequently, dependence of the respective values $C_{\Sigma}\left(t=t_{h}\right)$ on $x_{p}$ was calculated. As the panels (C), (D) show, larger $t_{h}$ leads to more pronounced transition in $C_{\Sigma}$. The entropy dependencies depicted in panels (E), (F) confirm the global trend which can be attributed to the enhanced selection due to higher $x_{p}$. We note, that this result was achieved presuming that all tumor clones are well identifiable which, however, contradicts to currently known limitations of biopsy and histology [71].

However, the slight global decrease in $x_{p}$ is not the only remarkable trend of the entropy as, apart from it, the local modulation caused by the presence of the threshold is present as well. For a more comprehensive understanding of the entropic dynamics, Fig 3.2 is presented, since projection of en- 
tropy values extracted exclusively for $t=t_{h}$ is not very illustrative Besides the plots of $S_{G}^{C}\left(t=t_{h}\right)$ and $S_{E P I G}^{C}\left(t=t_{h}\right)$, Fig 3.2 highlights the fact that entropy scenarios are universal owing to their similarity with those depicted in Fig.2.2

Interestingly, regarding the time dependencies $S_{G}^{C}(t)$ and $S_{E P I G}^{C}(t)$, both models, the quasispecies as well as the cancer population model, reveal many common and universal features, such as the overall single-peaked shape, direct proportions $S_{E P I G} \sim S_{G}$ and undulating $S_{E P I G}^{C}(t)$, which contrasts with much smoother $S_{G}^{C}(t)$. In addition, the findings imply that the amount of information/heterogeneity expressed by $S_{E P I G}^{C}$ exceeds $S_{G}^{C}$, which is consistent with the assumption of the additional epigenetic information [7], which warns that a "summative" framework [here represented by Eq.(3.11)] can overlook certain short-term details of the phenotypic population structure. Similarly as in the case of quasispecies model, the threshold effect emerges. In this case, its importance derives from its relevance to the therapeutic efficiency since the threshold separates growth regime from the regime of the slow growth or decay dynamics. Further details related to the investigation of the threshold sensitivity to the phase differences of the environment as well as supplementary algebraic characteristics derived for ODE system Eq. (3.9) are listed in the Appendix A.2

Fig $3.1(\mathrm{H})$ displays the formation of the asymptotic regime in the region above the threshold. As one can see, the following ascending ordering of the abundances stabilizes at long times $\left(t_{h} \geq 500\right)$ :

$$
C_{L 0}^{(0)}<C_{L 0}^{(1)}<C_{H}^{(0)}<C_{H}^{(1)}<C_{L 1}^{(1)}<C_{L 1}^{(0)} .
$$

The ordering at the level of the pairs $C_{L 0}^{(0)}<C_{L 0}^{(1)}$, $C_{H}^{(0)}<C_{H}^{(1)}, C_{L 1}^{(1)}<C_{L 1}^{(0)}$ is the consequence of imposed immunotherapeutic effects represented by $\alpha_{L}<\alpha_{H}$ involved in Eq. (3.6). Although design of $J_{s w H}$ is originally motivated to demonstrate superiority of reversible clones in the variable environments, our results show that pathway to drug resistance is not necessarily associated with the selective advantage of the clone $H$ (with the structure $[H ; z \in\{0,1\}])$. The situation, in which the abundances corresponding to $L 1$ exceed those of $H$ (which are more populated than $L 0$ ) is implied by the role of $\varphi_{L}$ and $\alpha_{H}$ (or $\alpha_{L}$ ) in variable environment. A qualitative explanation has origin in the structure of $J_{s w L 0}$ [see Eq.(3.5)]. According to it, the choice $\varphi_{L} \leq 1 / 2$ favors the phenotypic state $[L 0 ; z=0]$ compared to $[L 0 ; z=1]$. Similarly, due to net effect of $\varphi_{L}$, the structure of $J_{s w L 1}$ suggests commitment to $[L 1 ; z=0]$ thereby providing higher fit- ness than $[L 1 ; z=1]$. But the immunotherapeutic effect of $\alpha_{0}^{(0)}=\alpha_{H}$ is strong enough to cause a significant reduction of $C_{L 0}^{(0)}$. However, through evolution, the proliferative potential is redirected toward $C_{L 1}^{(0)}$, i.e. $[L 1 ; z=0]$, where cancer encounters only weakened immune reaction represented by $\alpha_{1}^{(0)}=\alpha_{L}$.

These qualitative explanations reflect complexity of the resistance-relevant processes and emphasize importance of computational effort to better understand their role in immunotherapeutic procedure. As a consequence, a single causal relationship between effects and the respective specific factor is not sufficient for complete understanding, as there is no single factor leading to the corresponding effect. The results show that the selective phenotypic advantage is formed due to a combined parametric effect comprising cellular response to drug specificity, phenotypic commitment, environmental dynamics, as well as other mechanisms included in the model.

\section{Discussion}

In the paper, we have proposed a minimalist concept of nonequilibrium modeling of the evolution of phenotypic reversibility in time-varying environments. The results demonstrate, at the methodological level, that the proposed ODE models can be helpful in understanding of the characteristic features of the evolutionary dynamics of genetic and epigenetic phenotypic states. At some stylized level, the space of alternatives is well described by the presented quasispecies and population models. As we have shown, a possible evolutionary process can be better understood when the structure of the underlying model involves combinations of low and high parametric values. In particular, the former of the two studied models, the quasispecies model, confirmed that under specific symmetric environmental conditions [where according to Eq. (2.1) the replication rates of phenotypic states move in symmetrically opposite directions $\left.d r^{(1)}(t)=-d r^{(0)}(t)\right]$ the clones with higher reversibility may outcompete their less reversible rivals, which is consistent with recent experimental findings [24]. Accordingly to both presented models, the high degree of reversibility represents an evolutionary advantage in the variable environments which disappears when the environments become static and vice versa.

Modeling of environmental changes proposed in this work is limited to stylized periodic variations. 
Further insights into the problem of variable environments can be derived for the stochastic models [72, 34, 73, 74, 52, 35, 36].

The model extension including more general asymmetric environmental influence may be laborious, but straightforward. It seems that ecology combining exogenous and endogenous environmental influence (where asymmetry can be a natural side product of the considerations) may be more important at the present stage of the modeling. In our stylized model of cancer cell population, the above abstract conceptualization is made more instructive by defining a periodic environment where exogenous variability of the effector cell population is considered.

Presented model of heterogeneous tumor growth (Section 3) demonstrates the model-specific therapeutic implications of evolved bet-hedging strategy and phenotypic plasticity of cancer cells. The levels of immunotherapeutic efficiency that cause remarkable differences between genetic and epigenetic entropy measures become prevalent at late simulation times, where mutation changes become very rare. Calculations show that effective therapies can be expected in the (stable) parametric area below a certain threshold of the parameter of therapeutic specificity. In difference to, e.g. the model [75], our model does not make a priori assumption of underlying drug resistance. On the other hand, the evolutionary viewpoint based on the concept of heterogeneity is a natural source of algebraic complications which presume more sophisticated theoretical treatment.

In general, many dynamic biosystems undergo a fundamental change of behavior when one of their parameters passes through a threshold value [76]. The concept of lethal threshold of unstable tumor progression has been discussed in [44, 45]. The identification of the threshold as a non-linear form of therapeutic effect on fitness is in line with the work [72]. In [77], a model of tumor growth is proposed where Allee threshold related to the minimum density criterion plays a central role. The ability to manipulate selectively the (micro)environment with the sustainable intensity and reliable detection of the threshold in the therapeutic efficiency could be major challenges for therapeutic applications, as exemplified may serve immunotherapeutic intervention [78]. The concept of environmental manipulation has been supported by experimental study [79] as well reporting that regular administration of drugs at nontoxic doses may overcome the drug resistance by shifting the therapeutic target from tumor cells to tumor endothelial cells. This therapeutic strategy is known under the term metronomic chemotherapy and immunotherapy. Regarding this, we put into attention one of the most findings from here presented cancer model, which is a positive therapeutic effect achieved with less (see also [47]) when moderate immunotherapeutic specificity (given by $\left.x_{p}\right)$ is assumed.

We note, that the original quasispecies model naturally led to the concept of "error threshold" for selective competence (see e.g. Fig.2.3 and [80]). Consequently, interesting question arises whether the threshold concept is transferable to therapeutic context as well.

Regarding the results of the threshold and sensitivity analysis (see A.2), the interesting question emerges whether carefully chosen linearized model classes which retain the typical evolutionary properties can be constructed and used to analyze the drug resistance observed in heterogeneous, evolving and variable cancer cell populations.

\section{Acknowledgments}

This work was supported by (a) APVV-15-0485 by Slovak Research and Development Agency; (b) VEGA No. 1/0250/18; (c) VEGA 1/0156/18 by Scientific Grant Agency of the Ministry of Education of Slovak Republic.

\section{A Appendices}

\section{A.1 Long run asymptotics - monoclonal fixation}

The quasispecies problem does not provide closedform expressions for the solution for a system of many species. Nevertheless, numerical results from Subsection 2.3 stimulated our interest in the asymptotic situation $t \gg 1 / \mu$ where the limit cycle

oscillations can be identified. We also found that for late-time evolution the single clone with the in$\operatorname{dex} n_{s} / 2$ is fixed. The case of highly localized (in $k$ ) species allows to consider more restrictive version of the constraint Eq. (2.6) in the form

$$
c\left(n_{s} / 2, t\right)=c^{(0)}\left(n_{s} / 2, t\right)+c^{(1)}\left(n_{s} / 2, t\right)=1 .
$$



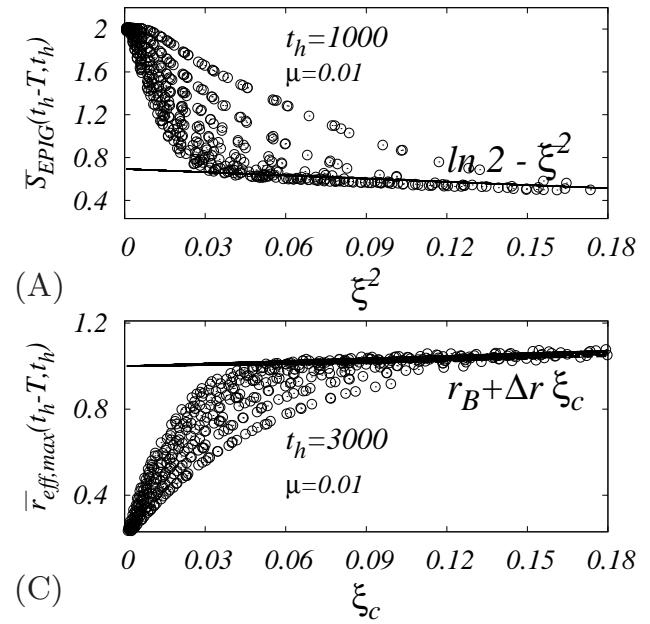
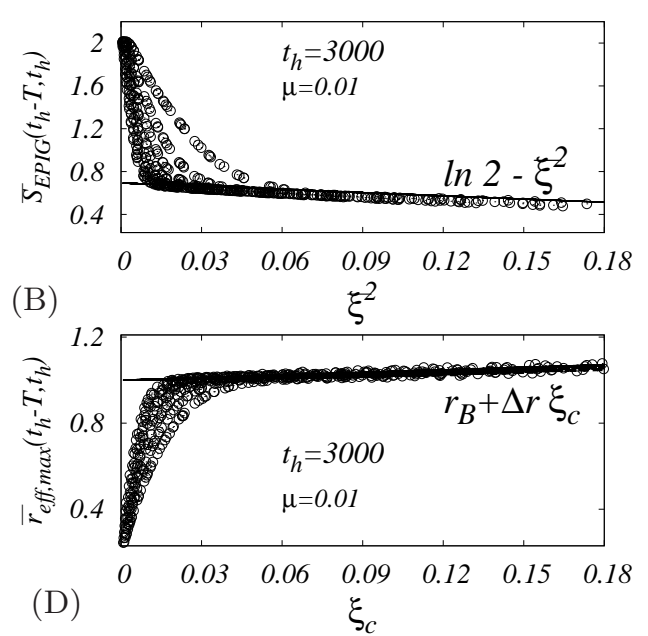

Figure 4.1: Figure depicts results of the sensitivity analysis focused on the role of $m, T, \Delta r$ parameters. The results were obtained for the time horizons $t_{h} \in\{1000,3000\}$ [see panels (A),(C) with $t_{h}=1000$; panels (B),(D) with $\left.t_{h}=3000\right]$ and constant $\mu=0.01$. Instead of particular dependencies on three parametric dimensions represented by $m, T, \Delta r$ (see Fig.2.3) (which lacks holistic perspective), we used $\overline{\xi^{2}}(m, T, \Delta r)$ (see Eq. (A.8) as a single independent variable. We have studied sensitivity on $\overline{\xi^{2}}(m, T, \Delta r)$ generated by the $(m, T, \Delta r)$ combinations with bounds $m \in\langle 0.01,0.2\rangle, T \in\langle 1,20\rangle, \Delta r \in\langle 0.1,0.4\rangle$. Since 8 evenly spaced values have been used for each particular variable, $8^{3}=512$ samples were drawn from the cubic hyper-lattice. Subsequently, each particular $(m, T, \Delta r)$ vector was converted to the $2 \mathrm{~d}$ projection consisting of the coordinates $\left[\bar{\xi}^{2}(m, T, \Delta r), \bar{S}_{E P I G}\left(t_{h}-T, t_{h}\right)\right]$, where $\bar{S}_{E P I G}\left(t_{h}-T, t_{h}\right) \stackrel{\text { def }}{=}(1 / T) \sum_{t \in\left\langle t_{h}-T, t_{h}\right\rangle} S_{E P I G}(t)$ [see panels (A), (B)]. Here, the summation is performed for the period $T$ preceding $t_{h}$. In analogy with Fig.(2.3), the thresholds reappear. When the plots (A), (B) are compared with the approximate form $\ln 2-\overline{\xi^{2}}$ (see Eq.A.11), particular agreement can be found for sufficiently high $\overline{\xi^{2}}$ (low entropy) region where selection process for the highest reversibility is more effective. The similar tendencies are exhibited by the mean replication measure $\bar{r}_{e f f, \max }\left(t_{h}-T, t_{h}\right) \stackrel{\text { def }}{=}(1 / T) \sum_{t \in\left\langle t_{h}-T, t_{h}\right\rangle} \max _{k=0, \ldots, n_{s}-1} r_{e f f}(k, t)$ [see panels (C), (D), where $\xi_{c}(m, T, \Delta r)$ defined by Eq. (A.7) plays a role analogous to that of $\overline{\xi^{2}}$; note that Eq. (2.15) is used to calculate $\left.r_{e f f}(k, t)\right]$. The long-time asymptotics $r_{B}+\Delta r \xi_{c}$ is given by Eq.A.10). In line with the expectations, the threshold is more pronounced and the relation look sharper at larger $t_{h}$ [panels(B), (D)].

It is consistent with two settings

$$
\begin{aligned}
& c_{\Sigma}^{(0)}(t) \simeq c^{(0)}\left(n_{s} / 2, t\right)=\frac{1}{2}+\xi(t), \\
& c_{\Sigma}^{(1)}(t) \simeq c^{(1)}\left(n_{s} / 2, t\right)=\frac{1}{2}-\xi(t),
\end{aligned}
$$

where the residual plasticity is transferred to the single auxiliary $-1 / 2<\xi(t)<1 / 2$. Consequently, using Eq.(2.3) we obtained $J_{s w}\left(n_{s} / 2, t\right)=-\xi$ and the equation for $\xi(t)$ can be written as

$$
\frac{d \xi}{d t}=\left(r_{0}-\Phi\right)\left(\frac{1}{2}+\xi\right)-m \xi .
$$

Substituting $c^{(0)}(t), \quad c^{(1)}(t)$ from Eq. A.2 into Eq.(2.15) we obtained

$$
\Phi(t) \simeq r_{e f f}(t)=r_{B}+2 \Delta r \xi(t) \cos \left(\frac{2 \pi t}{T}\right) .
$$

Its consequent substitution into Eq. (A.3) gives

$$
\frac{d \xi}{d t}=2 \Delta r\left(\frac{1}{4}-\xi^{2}\right) \cos \left(\frac{2 \pi t}{T}\right)-m \xi
$$

This nonlinear ODE problem can be solved using the single harmonic approximation (valid for $\left.\xi^{2} \ll 1 / 4\right)$ of the limit cycle

$$
\xi(t) \simeq \xi_{s} \sin \left(\frac{2 \pi t}{T}\right)+\xi_{c} \cos \left(\frac{2 \pi t}{T}\right)
$$

with the pair of amplitudes

$$
\xi_{s}=\frac{\pi T \Delta r}{4 \pi^{2}+T^{2} m^{2}}, \quad \xi_{c}=\frac{m T}{2 \pi} \xi_{s} .
$$

The formula clearly uncovers the relative effects of the processes operating at different time scales: $1 / \Delta r, 1 / m$ and $T$. The dependence upon $1 / \mu$ absents due to assumption that clonal selection has basically vanished in the long run. 
For the solutions given by Eq. A.6), the time averaging can be simply performed for the single period. The result is

$$
\overline{\xi^{2}} \simeq \frac{1}{2}\left(\xi_{s}^{2}+\xi_{c}^{2}\right) .
$$

Similarly, for $T$-periodic $\xi(t)$ the mean effective replication rate can be defined by

$$
\overline{r_{e f f}} \equiv \frac{1}{T} \int_{0}^{T} r_{e f f}(t) d t
$$

Then, using Eq.(2.15), Eq. (A.2), Eq.(A.4), Eq. A.6 and Eq.A.9 we obtained

$$
\overline{r_{e f f}}=r_{B}+\Delta r \xi_{c} .
$$

A posteriori confrontation with the condition $\xi^{2} \ll 1 / 4$ provides bounding $\Delta r \ll\left(4 \pi^{2}+\right.$ $\left.T^{2} m^{2}\right) /(\pi T \max \{1, m T\})$. Based on the structure of Eq.A.10, we have proposed the numerical analysis illustrated by examples in Appendix Fig 4.1 (C),(D) (see caption of this figure for more details). The combination with numerical tools in part justifies the use of asymptotic approximation presented in this Appendix.

We expect potential use of the result described by Eq. A.10 in increasing the efficiency of large-scale modeling, performed for the time intervals that are much longer than the period of the environment contained in the replication rate. A similar type of population-averaged growth rate is discussed in [54] within the context of gene expression of bacterial populations exposed to environmental variations.

Assuming that the only clone (the winner) survives, we get a trivial limit $\left.S_{G}\right|_{t \rightarrow \infty} \rightarrow 0$. However, the epigenetic alterations have non-trivial consequences. If Eq. A.2 is substituted into Eq.(2.10), one obtains

$$
S_{E P I G}(t) \simeq-\sum_{j \in\{-1,1\}}\left(\frac{1}{2}+j \xi(t)\right) \ln \left(\frac{1}{2}+j \xi(t)\right) .
$$

The formula can be analyzed by calculating its time averages. For this aim, the Taylor series of the order $\mathcal{O}\left(\xi^{2}\right)$ can be used. The entropy averaged over the period $\bar{S}_{E P I G} \simeq \ln 2-\overline{\xi^{2}}$ used in combination with Eq. A.7) and Eq. A.8 provides $\left(\bar{S}_{E P I G}-\right.$ $\ln 2) \sim\left(-\Delta r^{2}\right)$ in a qualitative agreement with the simulation trend shown in Fig.2.2(A),(D),(E),(F), Fig 2.3 B) and also short sensitivity study reported in Appendix Fig 4.1(A),(B).

It is clear that the calculations of the characteristics for separate variables are not universal across parametric values. The first step towards integrated view is manifested in Fig 4.1, where the time averaged $S_{E P I G}(t)$ and $\max _{k} r_{e f f}(k, t)$ are plotted versus auxiliary variables $\overline{\xi^{2}}$ and $\xi_{c}$ [see Eq.A.7) and Eq.A.8 below] for different combinations of $m, T, \Delta r$ inputs. The explicit forms of $\overline{\xi^{2}}(m, T, \Delta r)$ and $\xi_{c}(m, T, \Delta r)$ follow from the asymptotic results for the clone selection problem represented by Eq A.10

Contrary to previous considerations let us abandon for a moment predetermination of $\varphi\left(k_{\text {central }}\right)$. Instead, we discuss an alternative formulation that assumes equivalence of the asymptotic evolutionary solution with the one-dimensional static maximum of the mean $\overline{r_{e f f}}(\varphi)$. Such analysis is suitable for late times, when selection process is completed and, consequently, $k$-dependency of $\varphi(k)$ becomes irrelevant. In that case, the parametrization assuming the real-valued $\varphi \in\langle 0,1\rangle$ can be used and subsequent optimization performed.

In analogy with Eq. A.2 we assume $c^{(z)}(\varphi, t)=$ $1 / 2+(-1)^{z} \tilde{\xi}(\varphi, t)$. Continuous $\tilde{\xi}(\varphi, t)$ dependence may serve for the selection of the $\varphi_{\text {opt }} \in$ $\langle 0,1\rangle$ which solves the optimization task $\varphi_{\text {opt }}=$ $\arg \max _{\varphi \in\langle 0,1\rangle} \overline{\overline{r_{e f f}}}(\varphi)$. When $\varphi$ remains unspecified, the original Eq. A.5 may be rewritten to the form

$$
\frac{d \tilde{\xi}}{d t}=\frac{\Delta r}{2}\left(1-4 \tilde{\xi}^{2}\right) \cos \left(\frac{2 \pi t}{T}\right)+m\left(\frac{1}{2}-\tilde{\xi}-\varphi\right)(\mathrm{A}
$$

In analogy with Eq. A.4 we define $\widetilde{r_{e f f}}(\varphi, t)=r_{B}+$ $2 \Delta r \tilde{\xi} \cos (2 \pi t / T)$ and the corresponding time average $\overline{\widehat{r_{e f f}}}(\varphi)$. Obviously, the conflict between discrete $\varphi\left(k_{\text {central }}\right)$ and the solution of the continuous problem, $\varphi_{\text {opt }}$, can occur if the list of the possible discrete values $\{\varphi(k)\}_{k=0}^{n_{s}-1}$ does not contain $\varphi_{\text {opt }}$.

Nowadays, stochastic model variants become relatively independent field of research. For the lack of space, below we outline only an elementary model based on stochastic modification of the periodic environmental model. The reasons are as it follows: (i) recent studies point to the extrinsic noise as a factor influencing genetic and epigenetic determinants of the replication; (ii) there is an increased interest in a description of the intermediate environments between strictly periodic and noise processes; (iii) the noise responses can be considered as a suitable stability indicators.

Our elementary stochastic extension is based on the generalization in which the original regular $\cos (2 \pi t / T)$ function from Eq. A.12 is replaced by the periodized variant $w_{o u}(t)$ of the continuous Ornstein-Uhlenbeck random continuous process 
described by

$$
d w_{o u}=\frac{1}{\tau_{o u}}\left(\cos \left(\frac{2 \pi t}{T}\right)-w_{o u}\right)+\sigma_{o u} d W,
$$

where the constant $\sigma_{o u}$ changes the original unit dispersion of the standard Wiener process $W(t)$. The basic idea of the process is that relaxation to the instantaneous $\cos (2 \pi t / T)$ is described by the relaxation time $\tau_{o u}$. Moreover, as the periodization retains the influence of the former time scale $T$, different levels of stochasticity can be simulated using $\tau_{o u}$.

In accordance with the structure of Eq. A.12, new $w_{o u}(t)$ term is used to modify

$$
\begin{aligned}
\frac{d \xi_{o u}}{d t} & =\frac{\Delta r}{2}\left(1-4 \xi_{o u}^{2}\right) w_{o u}(t) \\
& +m\left(\frac{1}{2}-\xi_{o u}-\varphi\right)
\end{aligned}
$$

as well as the effective replication rate $r_{o u-e f f}=$
$r_{B}+2 \Delta r \xi_{o u} w_{o u}$. Again, to determine the optimum uniquely, the quantity $\overline{r_{\text {ou-eff }}}(\varphi)$ needs to be investigated for the late times where the effects of initial conditions become negligible.

As depicted in Fig A.1, the solution of the initial value problem obtained for given $\varphi$ can be used to calculate $\overline{\overline{r_{\text {ou-eff }}}}(\varphi)$. Since we are focussed on the region of $\tau_{o u} \ll T$ and very small $\sigma_{o u}$, there is no need for separate presentation related to deterministic limit. Two claims could be made regarding $w_{o u}, \xi_{o u}$ and $r_{o u-e f f}$ properties: (a) Because the relaxing of $w_{o u}(t)$ is unable to reach the heights of harmonic amplitudes, the process $\xi_{\text {ou }}(t)$ causes that $\overline{r_{\text {ou-eff }}}(\varphi)$ values are systematically smaller than their deterministic counterparts; (b) The fluctuations characterized by the variance $\operatorname{Var}\left(r_{\text {ou-eff }}(\varphi, t)-r_{B}\right)$ show an extremal impact of the highly unstable boundary regions $\varphi \rightarrow 0^{+}$and $\varphi \rightarrow 1^{-}$.
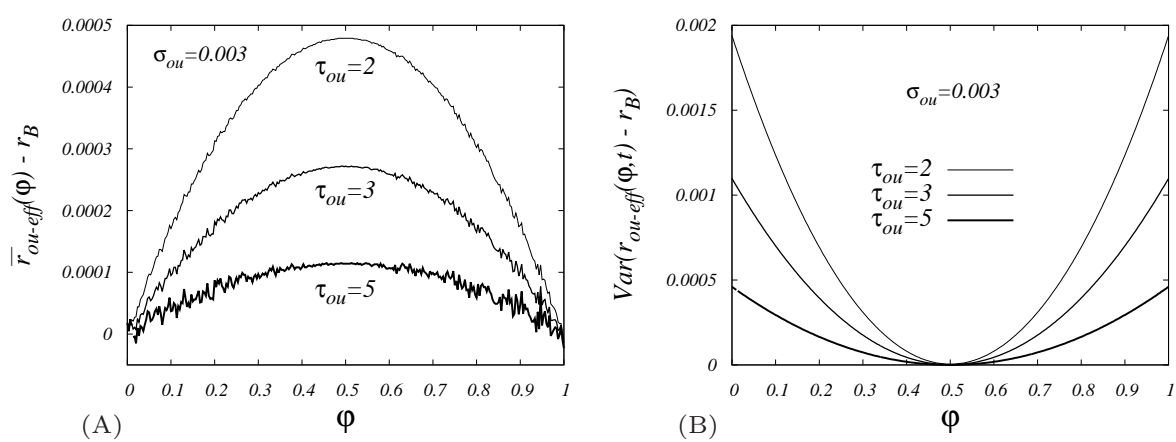

Figure A.1: The effective replication rate and corresponding fluctuations calculated for the parameters $T=$ $10, \Delta r=0.1, m=0.1$ using Eq. A.14). To attain stationary regime, initial 200 periods were discarded. Next thousand periods were used to calculate the mean values. Panel A confirms the optimality of the mean clonal replication for $\varphi=1 / 2$ is confirmed. According to panel (B), the largest fluctuations are observed for the boundary region.

\section{A.2 Therapeutic threshold, initial stages of tumor formation, sen- sitivity analysis}

To conceive better the effect of the therapeutic threshold as well as to support generality of the suggested concept (for $x_{p}$ parameter), we analyze initial stages of the tumor formation in more detail. Additionally to our previous layout, below we focus on the sensitivity analysis in the space of therapeutic options or available uncertain inputs. For that aim, we formulate the threshold problem in a way enabling us to carry out suitable analytical or semi-analytical approximations.

Although the issues of uncertainty and risk are not typically addressed within the ODE, the relevant information in this context can be obtained by studying assembly of alternative phase shifts that are exogenous inputs of our ODE system. Assuming that medical treatment will be confronted with an uncertainty in the abundance of the effector cell population $(\sim \Delta e)$, the additional phase variability has been incorporated by modifying the original periodic model of $e(t)$. In the modified version, for every simulation run $j=1,2, \ldots, N_{e}$ of the duration $t_{h}$ the specific $j$-dependent phase shift $2 \pi j / N_{e}$ is introduced as an extra degree of freedom with the corresponding abundance

$$
e_{j}(t)=e_{B}+\Delta e \cos \left[2 \pi\left(\frac{j}{N_{e}}+\frac{t}{T}\right)\right]
$$



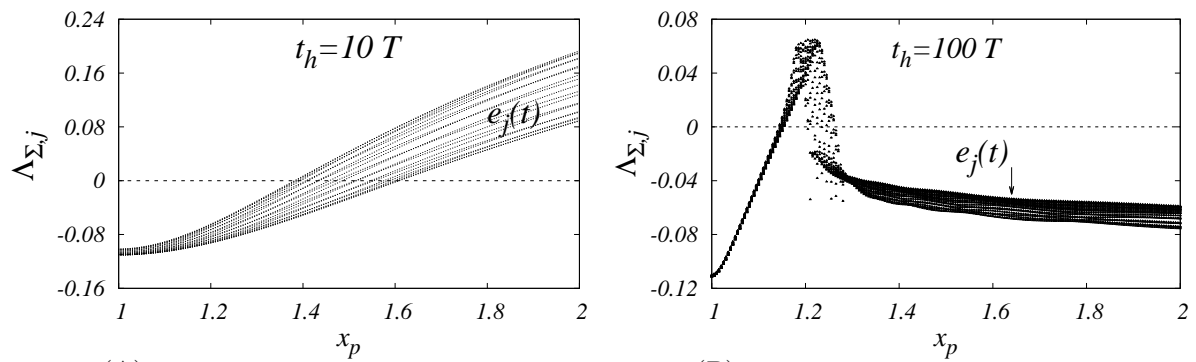

(A)

(B)
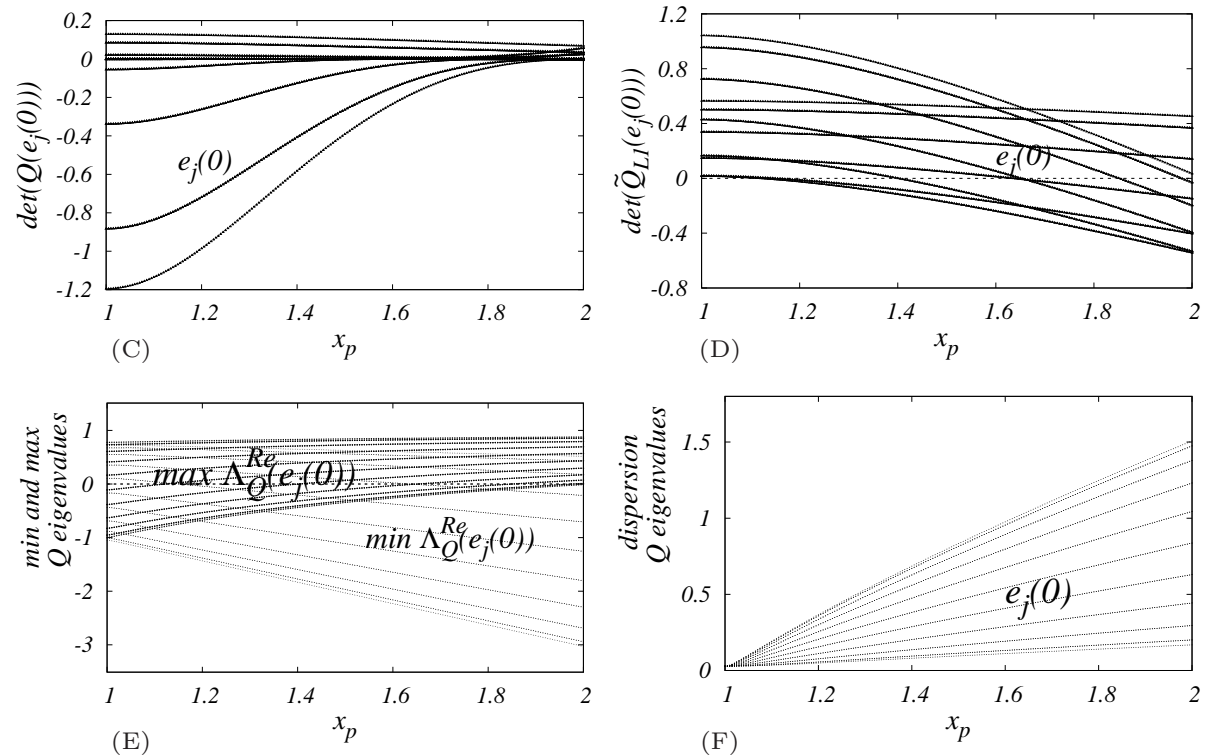

Figure A.2: Auxiliary calculations that allow us to better understand the complexities around the threshold of the parameter $x_{p}$. Calculations, as in many previous cases, were performed for parameters $a=1, r=1$, $m=0.05, \mu=0.01, T=10, g=0.45, e_{B}=0.5, \Delta e=0.4, \varphi_{L}=0.25, C_{P 0}=0.001$. The new parameter $\delta C_{P 0}=0.0001$ [see Eq. A.17] characterizes initial separation of trajectories; $N_{e}=20$ variants of the phase shifted $e_{j}(t)$ and $e_{j}(0)$ have been taken into account to characterize the sensitivity. Panels (A), (B) show how the growth instability is manifested by the Lyapunov-like exponents $\Lambda_{\Sigma, j}, j=1,2, \ldots, N_{e}$. We see that the influence of phase shifts is getting weaker as the time is passing [shadowing of initial condition Eq.(3.12) where the sole nonzero abundance is $\left.C_{P}(0)\right]$. For the purposes of alternative approach, we consider panels $(\mathrm{C})$ and $(\mathrm{D})$ including $\operatorname{det}\left(Q\left(e_{j}(0)\right)\right)$ (as a product of eigenvalues) and $\operatorname{det}\left(\tilde{Q}_{L 1}\left(e_{j}(0)\right)\right)$, respectively. Interestingly, there are many mutual crossings in (D) starting above $x_{p} \sim 1.2$. Panel (E) shows maximal and minimal elements of the set $\Lambda_{Q}^{R e}\left(e_{j}(0)\right)$ (determined for each $e_{j}(0)$ separately). Alternatively, the system of dispersions in panel (F) helps us to characterize the distribution of eigenvalues arranged in $\Lambda_{Q}^{R e}\left(e_{j}(0)\right)$. 
on the interval $t \in\left\langle 0, t_{h}\right\rangle$. In such case, dynamics of $e_{j}(t)$ influences the respective population dynamics through $\propto-\tilde{\alpha} a e_{j}(t) \tilde{C} /\left(C_{\Sigma, j}+g\right)$ term modifying the original form of Eq. (3.6). The sensitivity to the phase differences provide additional information related to the system trajectories.

We assume that within the context of here studied growth phenomena, the relevant information can be described by the Lyapunov-like exponent

$$
\Lambda_{\Sigma, j}=\frac{1}{t_{h}} \ln \frac{\left|C_{\sum, j}^{[1]}\left(t_{h}\right)-C_{\Sigma, j}^{[2]}\left(t_{h}\right)\right|}{\left|\delta C_{P 0}\right|} .
$$

defined by means of two horizon values $C_{\Sigma, j}^{[1]}\left(t_{h}\right)$, $C_{\Sigma, j}^{[2]}\left(t_{h}\right)$ [splitting the original $C_{\Sigma}$ from Eq. (3.4)] obtained under the action of $e_{j}(t)$ for two initial conditions

$$
\begin{aligned}
& \text { path } C_{\Sigma, j}^{[1]}(t): \text { initial } C_{P}(0)=C_{P 0}, \quad(\mathrm{~A} .17) \\
& \text { path } C_{\Sigma, j}^{[2]}(t): \text { initial } C_{P}(0)=C_{P 0}+\delta C_{P 0},
\end{aligned}
$$

where $\delta C_{P 0}$ is small shift in $C_{P 0}$ defined by Eq. 3.12).

Since the system of phenotypes begins to evolve from a small monoclonal primary tumor, there is no initial heterogeneity (see Fig 2.2 and Fig.3.2. Thus, for small times the dynamics of species abundances allows for a linear analysis that offers some computational benefits. This section continues with the development of semi-analytical approximation which supports relevance of the threshold approach. The aim is to extend this intuitive concept by examining stability of the initial conditions quantified by the spectrum of the local Lyapunov exponents.

Within the applied quasi-static approximation each $e_{j}(t), j=1,2, \ldots, N_{e}$ is replaced by the constant value $e_{j}(0)=e_{B}+\Delta e \cos \left(2 \pi j / N_{e}\right)$. In such elementary case, Eq. (3.9) can be linearized in the vicinity of the initial condition given by Eq.(3.12). The linearization provides an approximate solution with the dynamic "fastest" mode written in the matrix form $\exp \left(\mathbf{Q}\left(e_{j}(0)\right) t\right)$, where $\mathbf{Q}\left(e_{j}(0)\right)$ denotes the local Jacobian matrix of the system Eq.(3.9) we specify in the following. Then, the diagonalization of $\mathbf{Q}\left(e_{j}(0)\right)$ can be performed and the stability of the initial condition can be checked by investigating real parts of eigenvalues

$$
\Lambda_{Q, j}^{R e} \equiv \operatorname{Re}\left[\operatorname{diag}\left(\mathbf{Q}\left(e_{j}(0)\right)\right]\right.
$$

Then, by studying $N_{e}$ variants of $\Lambda_{Q, j}^{R e}$ spectra the possible impacts of the therapy on the very early stages of tumor formation can be obtained.
For later purposes, we introduce auxiliary variables

$$
\begin{aligned}
r_{P 0} & =r\left(1-C_{P 0}\right), a_{e}=\frac{a e_{j}(0) g}{\left(C_{P 0}+g\right)^{2}}, \\
Q_{P} & =r_{P 0}-r C_{P 0}-a_{e}-6 \mu, \\
Q_{a e} & =\left(a_{e}-r\right) C_{P 0} .
\end{aligned}
$$

The resulting $\mathbf{Q}$ can be ordered into the blocks

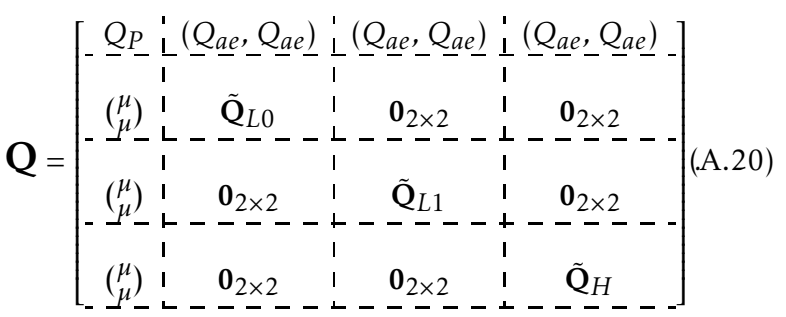

Here, the evolutionary mechanism is highlighted by the leftmost column which mediates the interconnection of species. The first row including nondiagonal $Q_{a e}$ elements simply reflects the immune feedback. Three $2 \times 2$ sub-matrices of the type

$$
\tilde{\mathbf{Q}}_{X}=\left(\begin{array}{cc}
Q_{X}^{(0)} & m\left(1-\varphi_{L}\right) \\
m \varphi_{L} & Q_{X}^{(1)}
\end{array}\right), X \in\{L 0, L 1, H\}
$$

correspond to the respective clones $L 0, L 1, H$. Their switching capability is evident from the nondiagonal structure of $\tilde{\mathbf{Q}}_{X}$. The interplay of the proliferative, switching, immune and therapeutic effects is compactly expressed in the following diagonal terms $Q_{X}^{(z)}, z \in\{0,1\}$. For the phenotypic alternative $z=0$ we have

$$
\left(\begin{array}{c}
Q_{L 0}^{(0)} \\
Q_{L 1}^{(0)} \\
Q_{H}^{(0)}
\end{array}\right)=r_{P 0}-m\left(\begin{array}{c}
\varphi_{L} \\
\varphi_{L} \\
\frac{1}{2}
\end{array}\right)-\frac{a e_{j}(0)}{C_{P 0}+g}\left(\begin{array}{c}
x_{p} \\
\frac{1}{x_{p}} \\
x_{p}
\end{array}\right),
$$

whereas in the $z=1$ case we obtained

$$
\left(\begin{array}{c}
Q_{L 0}^{(1)} \\
Q_{L 1}^{(1)} \\
Q_{H}^{(1)}
\end{array}\right)=r_{P 0}-m\left(\begin{array}{c}
1-\varphi_{L} \\
1-\varphi_{L} \\
\frac{1}{2}
\end{array}\right)-\frac{a e_{j}(0)}{C_{P 0}+g}\left(\begin{array}{c}
\frac{1}{x_{p}} \\
x_{p} \\
\frac{1}{x_{p}}
\end{array}\right)
$$

The methodology offers interpretation in which the threshold of $x_{p}$ separates the phase of small and large instability. The results of numerical calculations are shown in Fig A.2 Important general finding is, that the threshold behavior can be manifested not only by the nonlocal measures (e.g. using $\Lambda_{\Sigma, j}$ ), but by the calculations based on $\mathbf{Q}\left(e_{j}(0)\right)$ as well. The long-term $\left(t_{h}=100 T\right)$ dependence $\Lambda_{\Sigma, j}\left(x_{p}\right)$ depicted in Fig A.2 (B) seems to be fairly consistent with roughly estimated threshold $x_{p, \text { thr }} \in(1.12,1.14)$ identifiable from Fig $3.1(\mathrm{H})$. In addition, regarding sensitivity to $e_{j}(t)$, Fig A.2(B) shows that after a long period, the consequences 
of the phase differences gradually vanish due to convergence onto the universal (in the sense of its phase-independence) limit cycle attractor. Conversely, the panel $(\mathrm{A})$, where $t_{h}=10 \mathrm{~T}$, shows no more evidence for the threshold-type behavior in the terms $\Lambda_{\Sigma, j}$. Regarding evaluation of the set of the measures derived from $\mathbf{Q}\left(e_{j}(0)\right)$, it seems that detailed enough knowledge available from the local characteristics derived from $\mathbf{Q}\left(e_{j}(0)\right)$ makes it possible to substitute the lack of information.

The local characteristics based on $\mathbf{Q}\left(e_{j}(0)\right)$ show capacity to unmask expanding or contracting ten- dencies that vary with $x_{p}$. While $\operatorname{det}\left(\mathbf{Q}\left(e_{j}(0)\right)\right)$ depicted in the panels (C), (D) indicates that system of trajectories may begin to expand due to $x_{p}$, the minors $\operatorname{det}\left(\mathbf{Q}_{L 1}\left(e_{j}(0)\right)\right), j=1,2, \ldots, N_{e}$, on the contrary, indicate a shift towards more contractive effects (we verified that minors $\operatorname{det}\left(\tilde{\mathbf{Q}}_{L 0}\right)$ and $\operatorname{det}\left(\tilde{\mathbf{Q}}_{H}\right)$ confirm the same tendency). It means, that therapeutically induced separation of eigenvalues $\Lambda_{Q, j}^{R e}$ [see panels (E), (F) of Fig A.2] cannot be simply attributed to diversity of subpopulations, but, more likely, it reflects diversity of the replication rates causing the rapid clonal expansion with possible dominance of fittest species. 


\section{References}

[1] P. C. Nowell. The clonal evolution of tumor cell populations. Science, 194:23-28, 1976.

[2] L. M. F. Merlo, J. W. Pepper, B. J. Reid, and C. C. Maley. Cancer as an evolutionary and ecological process. Nat. Rev. Cancer, 6:924935, 2006.

[3] M. Greaves. Darwinian medicine: a case for cancer. Nat. Rev. Cancer, 7:213-221, 2007.

[4] R. J. Gillies, D. Verduzo, and R. A. Gatenby. Evolutionary dynamics of carcinogenesis and why targeted therapy does not work. Nat. Rev. Cancer, 12:487-493, 2012.

[5] P. W. Laird. Cancer epigenetics. Hum. Mol. Gen., 14:R65-R76, 2005.

[6] A. Willbanks, M. Leary, M. Greenshields ansd C. Tyminski, S. Heerboth, K. Lapinska, K. Haskins, and S. Sarkar. The evolution of epigenetics: From prokaryotes to humans and its biological consequences. Genet Epigenet, 8:25-36, 2016.

[7] H. T. Bjornsson, M. D. Fallin, and A. P. Feinberg. An integrated epigenetic and genetic approach to common human disease. Trends Genet., 20:350-358, 2004.

[8] H. Easwaran, H. C. Tsai, and S. B. Baylin. Cancer epigenetics: Tumor heterogeneity, plasticity of stem-like states, and drug resistance. Mol Cell, 54:716-727, 2014.

[9] M. Greaves. Evolutionary Determinants of Cancer. Cancer Discov., 5:806-820, 2015.

[10] O. Carja and J.B. Plotkin. The evolutionary advantage of heritable phenotypic heterogeneity. Sci. Rep., 7:5090, 2017.

[11] S. Huang. Genetic and non-genetic instability in tumor progression: link between the fitness landscape and the epigenetic landscape of cancer cells. Cancer Metastasis Rev., 32:423448, 2013.

[12] O. J. Rando and K. J. Verstrepen. Timescales of genetic and epigenetic inheritance. Cell, 128:655-668, 2007.

[13] A. J. Crean and D. J. Marshall. Coping with environmental uncertainty: dynamic bet hedging as a maternal effect. Phil. Trans. R. Soc. B, 364:1087-1096, 2009.
[14] S. Forbes. Portfolio theory and how parent birds manage investment risk. Oikos, 118:1561-1569, 2009.

[15] H. J. E. Beaumont, J. Gallie, C. Kost, G. C. Ferguson, and P. B. Rainey. Experimental evolution of bet hedging. Nature, 462:90-94, 2009.

[16] I. de Jong, P. Haccou, and O. P. Kuipers. Bet hedging or not? A guide to proper classification of microbial survival strategies. BioEssays, 33:215-223, 2011.

[17] M. C. Donaldson-Matasci, M. Lachmann, and C. T. Bergstrom. Phenotypic diversity as an adaptation to environmental uncertainty. Evol Ecol Res., 10:493-515, 2008.

[18] B. Brutovsky and D. Horvath. Structure of intratumor heterogeneity: Is cancer hedging its bets? arXiv.org, abs/1307.0607, 2013.

[19] D. Nichol, M. Robertson-Tessi, P. Jeavons, and A. R. A. Anderson. Stochasticity in the genotype-phenotype map: Implications for the robustness and persistence of bethedging. Genetics, 204:1523-1539, 2016.

[20] D. Horvath and B. Brutovsky. Etiology of phenotype switching strategies in time varying environment. Phys. Lett. A, 380(13):1267$1278,2016$.

[21] C. A. Gravenmier, M. Siddique, and R. A. Gatenby. Adaptation to stochastic temporal variations in intratumoral blood flow: The warburg effect as a bet hedging strategy. Bull Math Biol., 2017.

[22] F. Thomas, B. Ujvari, C. Gideon, A. Tasiemski, P. W. Ewald, and B. Roche. Toward an ultimate explanation of intratumor heterogeneity. In B. Ujvari, B. Roche, and F. Thomas, editors, Ecology and evolution of cancer. Elsevier, 2017.

[23] R. H. Chisholm, T. Lorenzi, and J. Clairambault. Cell population heterogeneity and evolution towards drug resistance in cancer: Biological and mathematical assessment, theoretical treatment optimisation. Biochim Biophys Acta, 1860:2627-2645, 2016.

[24] R.A. Mathis, E.S. Sokol, and P.B. Gupta. Cancer cells exhibit clonal diversity in phenotypic plasticity. Open Biol., 7(2), 2017.

[25] K. Kemper, P. L. de Goeje, D. S. Peeper, and R. van Amerongen. Phenotype switching: Tumor cell plasticity as a resistance mechanism 
and target for therapy. Cancer Res., 74:59375941, 2014.

[26] M. S. Rogers, K. Novak, D. Zurakowski, L. M. Cryan, Anna Blois, E. Lifshits, T. H. Bø, A. M. Oyan, E. R. Bender, Michael Lampa, Soo-Young Kang, K. Naxerova, K.-H. Kalland, O. Straume, L. A. Akslen, R. S. Watnick, J. Folkman, and G. N. Naumov. Spontaneous reversion of the angiogenic phenotype to a nonangiogenic and dormant state in human tumors. Mol. Cancer Res., 12:754-764, 2014.

[27] D. Klevebring, G. Rosin, R. Ma, J. Lindberg, K. Czene, J. Kere, I. Fredriksson, J. Bergh, and J. Hartman. Sequencing of breast cancer stem cell populations indicates a dynamic conversion between differentiation states in vivo. Breast Cancer Res., 16:R72, 2014.

[28] A. O. Pisco and S. Huang. Non-genetic cancer cell plasticity and therapy-induced stemness in tumour relapse: 'What does not kill me strengthens me'. Br. J. Cancer, 112:17251732, 2015.

[29] M. F. Emmons, F. Faião-Flores, and K. S. M. Smalley. The role of phenotypic plasticity in the escape of cancer cells from targeted therapy. Biochem. Pharmacol., 122:1-9, 2016.

[30] S. Liu, F. Zhou, Y. Shen, Y. Zhang, H. Yin, Y. Zeng, J. Liu, Z. Yan, and X. Liu. Fluid shear stress induces epithelial-mesenchymal transition (emt) in Hep-2 cells. Oncotarget, 7:32768-32892, 2016.

[31] M. Saez-Ayala, M. F. Montenegro, L. Sanchez del Campo, M. P. Fernandez-Perez, S. Chazarra, R. Freter, M. Middleton, A. PineroMadrona, J. Cabezas-Herrera, C. R. Goding, and J. N. Rodrguez-Lopez. Directed phenotype switching as an effective antimelanoma strategy. Cancer Cell, 24:105-119, 2013.

[32] T. Germain, M. Ansari, and D. Pappas. Observation of reversible, rapid changes in drug susceptibility of hypoxic tumor cells in a microfluidic device. Anal. Chim. Acta, 936:179184, 2016.

[33] V. K. Mishra and S. A. Johnsen. Targeted therapy of epigenomic regulatory mechanisms controlling the epithelial to mesenchymal transition during tumor progression. Cell Tissue Res., 356:R617-630, 2014.
[34] O. Carja and M. W. Feldman. An equilibrium for phenotypic variance in fluctuating environments owing to epigenetics. J. R. Soc. Interface, 9:613-623, 2012.

[35] X.-D. Zheng, C. Li, S. Lessard, and Y. Tao. Evolutionary stability concepts in a stochastic environment. Phys. Rev. E, 96:032414, 2017.

[36] K. Wienand, E. Frey, and M. Mobilia. Evolution of a fluctuating population in a randomly switching environment. Phys. Rev. Lett., 119:158301, 2017.

[37] J. J. Herman, H. G. Spencer, K. Donohue, and S. E. Sultan. How stable 'should' epigenetic modifications be? Insights from adaptive plasticity and bet-hedging. Evolution, 68:632-643, 2014.

[38] M. Eigen, J. McCaskill, and P. Schuster. The molecular quasi-species. Adv. Chem. Phys., 75:149-263, 1989.

[39] E. Baake, M. Baake, and H. Wagner. Ising quantum chain is equivalent to a model of biological evolution. Phys. Rev. Lett., 78:559$562,1997$.

[40] F. Bagnoli and M. Bezzi. Eigens error threshold and mutational meltdown in a quasispecies model. Int. J. Mod. Phys. C, 09(07):9991005, 1998.

[41] K. Malarz and D. Tiggemann. Dynamics in eigen quasispecies model. Int. J. Mod. Phys. C, 09(03):481-490, 1998.

[42] G. Bianconi and Ch. Rahmede. Unified framework for quasispecies evolution and stochastic quantization. Phys. Rev. E, 83:056104, 2011.

[43] C. O. Wilke. Quasispecies theory in the context of population genetics. BMC Evol. Biol., 5:44, 2005.

[44] D.R. Amor and R.V. Solé. Catastrophic shifts and lethal thresholds in a propagating front model of unstable tumor progression. Phys. Rev. E, 90:022710, 2014.

[45] J. Sardanyé, R. Martínez, C. Simó, and R. Solé. Abrupt transitions to tumor extinction: a phenotypic quasispecies model. J. Math. Biol., 74(7):1589-1609, 2017.

[46] C. C. Maley, A. Aktipis, T. A. Graham, A. Sottoriva, A. M. Boddy, M. Janiszewska, A. S. Silva, M. Gerlinger, Y. Yuan, K. J. Pienta, K. S. 
Anderson, R. Gatenby, C. Swanton, D. Posada, C. I. Wu, J. D. Schiffman, E. Shelley Hwang, K. Polyak, A. R. A. Anderson, J. S. Brown, M. Greaves 4, and D. Shibata. Classifying the evolutionary and ecological features of neoplasms. Nat. Rev. Cancer, 17:605-619, 2017.

[47] A. Lorz, T. Lorenzi, J. Clairambault, A. Escargueil, and B. Perthame. Modeling the effects of space structure and combination therapies on phenotypic heterogeneity and drug resistance in solid tumors. Bull Math Biol., 77:122, 2015.

[48] D. Horvath and G. Kneller. A leastconstraint principle for population dynamics and reaction kinetics: Modeling entropycontrolled chemical hypercycles. J Chem Phys, 131(17):171101, 2009.

[49] D. Horvath, B. Brutovsky, J. Kocisova, and S.Sprinc. Manipulation with heterogeneity within a species population formulated as an inverse problem. Physica A, 389(21):50285036, 2010.

[50] G. C. Urtel, T. Rind, and D. Braun. Reversible switching of cooperating replicators. Phys. Rev. Lett., 118:078102, 2017.

[51] R. A. Cairns, T. Kalliomaki, and R.P. Hill. Acute (Cyclic) Hypoxia Enhances Spontaneous Metastasis of KHT Murine Tumors. Cancer Res., 61(24):8903-8908, 2001.

[52] T.J. Kobayashi and Y. Sughiyama. Stochastic and information-thermodynamic structures of population dynamics in a fluctuating environment. Phys. Rev. E, 96:012402, 2017.

[53] S. Fedotov and A. Iomin. Migration and proliferation dichotomy in tumor-cell invasion. Phys. Rev. Lett., 98:118101, 2007.

[54] M. Thattai and A. van Oudenaarden. Stochastic gene expression in fluctuating environments. Genetics, 167:523-530, 2004.

[55] O. Rivoire and S. Leibler. The value of information for populations in varying environments. J Stat Phys, 142:1124-1166, 2011.

[56] M. E. Palmer and M. W. Feldman. Survivability is more fundamental than evolvability. PLoS ONE, 7:e38025, 2012.

[57] E. Kussell and S. Leibler. Phenotypic diversity, population growth, and information in fluctuating environments. Science, 309:20752078, 2005.
[58] L. Dercle, S. Ammari, M. Bateson, P. B. Durand, E. Haspinger, Ch.Massard, C. Jaudet, A. Varga, E. Deutsch, J.-Ch. Soria, and Ch. Ferte. Limits of radiomic-based entropy as a surrogate of tumor heterogeneity: Roi-area, acquisition protocol and tissue site exert substantial influence. Sci. rep., 7(1):7952, 2017.

[59] D. Kirschner and J. C. Panetta. Modeling immunotherapy of the tumor-immune interaction. J. Math. Biol, 37(3):235-252, 1998.

[60] A. Talkington, C. Dantoin, and R. Durrett. Ordinary differential equation models for adoptive immunotherapy. Bull Math Biol., 2017.

[61] A. Tsygvintsev, M. Simeone, and D.E. Kirschner. A Mathematical Model of Gene Therapy for the Treatment of Cancer. Springer, New York, 2013.

[62] E. Piretto, M. Delitala, and M. Ferraro. Combination therapies and intra-tumoral competition: Insights from mathematical modeling. J Theor Biol, 446:149-159, 2018.

[63] A. d'Onofrio, A. Gandolfi, and S. Gattoni. The norton-simon hypothesis and the onset of non-genetic resistance to chemotherapy induced by stochastic fluctuations. Physica A, 391:6484-6496, 2012.

[64] A. d'Onofrio, F. Gatti, P. Cerrai, and L. Freschi. Delay-induced oscillatory dynamics of tumourimmune system interaction. Mathematical and Computer Modelling, 51:572-591, 2010.

[65] R. A. Gatenby and T. L. Vincent. An evolutionary model of carcinogenesis. Cancer Research, 63(19):6212-6220, 2003.

[66] P. Gerlee and A. R. A. Anderson. The evolution of carrying capacity in constrained and expanding tumour cell populations. Physical Biology, 12(5):056001, 2015.

[67] D. Liao, L. Estevez-Salmeron, and T.D. Tlsty. Conceptualizing a tool to optimize therapy based on dynamic heterogeneity. Phys Biol., 9(6):065005, 2012.

[68] E. Kussell, R. Kishony, N. Q. Balaban, and S. Leibler. Bacterial persistence: A model of survival in changing environments. Genetics, 169:1807-1814, 2005.

[69] E. Libby and P. B. Rainey. Exclusion rules, bottlenecks and the evolution of stochastic phenotype switching. Proc. R. Soc. Lond. B, 278:3574-3583, 2011. 
[70] S. Ilyas and J.C. Yang. Landscape of tumor antigens in t cell immunotherapy. J Immunol., 195(11):5117-5122, 2015.

[71] M. Gerlinger, A.J. Rowan, and et al. S. Horswell. Intratumor heterogeneity and branched evolution revealed by multiregion sequencing. N Engl J Med., 366(10):883-892, 2012.

[72] D.A. Charlebois, N. Abdennur, and M. Kaern. Gene expression noise facilitates adaptation and drug resistance independently of mutation. Phys. Rev. Lett., 107:218101, 2011.

[73] B.E. Saether and S. Engen. The concept of fitness in fluctuating environments. Trends Ecol Evol., 30:273-281, 2015.

[74] P.G. Hufton, Y.T. Lin, and T.Galla. Phenotypic switching of populations of cells in a stochastic environment. Journal of Statistical Mechanics: Theory and Experiment, 2018(2):023501, 2018.
[75] P. Greulich, B. Waclaw, and R.J. Allen. Mutational pathway determines whether drug gradients accelerate evolution of drug-resistant cells. Phys. Rev. Lett., 109:088101, 2012.

[76] R. Solé, D. R. Amor, S. D. Nebreda, N. C. Pueyo, M. C. Ballestero, and R. Montanez. Synthetic collective intelligence. In Biosystems, 148:47-61, 2016.

[77] L. Sewalt, K. Harley, P. van Heijster, and S. Balasuriya. Influences of allee effects in the spreading of malignant tumours. J. Theor. Biol., 394(Supp. C):77 - 92, 2016.

[78] Z. Li, L. Chen, and M.P. Rubinstein. Cancer immunotherapy: are we there yet? Exp. Hematol. Oncol., 2, 2013.

[79] M.Ch. Chang Yu-Li Chen and W.F.Cheng. Metronomic chemotherapy and immunotherapy in cancer treatment. Cancer Lett., 400:282-292, 2017.

[80] C.K. Biebricher and M. Eigen. The error threshold. Virus Res., 107(2):117-127, 2005. 